\title{
The active zone protein Clarinet regulates ATG-9 trafficking at synapses and presynaptic autophagy
}

3

4

\section{Affiliations:}

${ }^{1}$ Program in Cellular Neuroscience, Neurodegeneration and Repair, Departments of Neuroscience and Cell Biology, Yale University School of Medicine, 260 Whitney Avenue, YSB C167, New Haven, CT 06511 ${ }^{2}$ Instituto de Neurobiología, Recinto de Ciencias Médicas, Universidad de Puerto Rico, 201 Boulevard del Valle, San Juan 00901, Puerto Rico

\# Equal contribution

${ }^{*}$ Correspondence to:

Daniel A. Colón-Ramos, Ph.D.

Department of Neuroscience

Department of Cell Biology

Yale University School of Medicine

260 Whitney Avenue

YSB C167

New Haven, CT 06511

Email: daniel.colon-ramos@yale.edu 


\section{Highlights}

- The long isoform of the active zone protein Clarinet (CLA-1L) regulates ATG-9 trafficking at synapses

- CLA-1L extends from the active zone to the periactive zone and cooperates with the periactive zone endocytic proteins EHS-1/EPS15 and ITSN-1/ intersectin 1 in ATG-9 trafficking during exo-endocytosis

- Mutations in CLA-1L, or in clathrin-associated adaptor molecules, result in abnormal accumulation of ATG-9 into clathrin-rich endocytic intermediates

- CLA-1L mutants which affect ATG-9 trafficking are also defective in activitydependent presynaptic autophagy

60

61 Keywords

62 Autophagy, Clarinet, active zone proteins, periactive zone, endocytosis, ATG-9, 63 AP-1, AP-2

64 


\section{Abstract}

In neurons, autophagy is temporally and spatially regulated to occur near

68 presynaptic sites. How trafficking of autophagy proteins is regulated to support

69 synaptic autophagy is not well understood. From forward genetic screens, we

70 identify a role for the long isoform of the active zone protein Clarinet (CLA-1L) in

71 regulating trafficking of autophagy protein ATG-9 at synapses, and presynaptic

72 autophagy. ATG-9 is a transmembrane protein that undergoes activity-dependent

73 exo-endocytosis at synapses, and mutations in CLA-1L result in abnormal

74 accumulation of ATG-9 into clathrin-rich endocytic intermediates. CLA-1L extends

75 from the active zone to the periactive zone, and genetically interacts with periactive

76 zone proteins required for clathrin-dependent endocytosis. We find that CLA-1L is

77 specifically required for sorting of ATG-9 at synapses, likely via endosome-

78 mediated endocytosis, and for activity-dependent presynaptic autophagy. Our

79 findings provide mechanistic insights into how active zone proteins regulate key

80 steps of ATG-9 exo-endocytosis, a process that could couple the activity state of

81 the neuron and autophagy.

82 


\section{Introduction}

84 Macroautophagy (herein called autophagy) is a well conserved cellular

85 degradative pathway, and its disruption in neurons result in axonal degeneration,

86 accumulation of protein aggregates and cell death ${ }^{1-4}$. Neuronal autophagy is

87 regulated to cater to the particular physiological needs of neurons ${ }^{5,6}$. Regulated

88 steps in neurons include temporal and spatial control of autophagosome

89 biogenesis near synaptic sites and in response to increased neuronal activity

90 states $^{7-18}$. The spatial regulation of autophagosome biogenesis at synapses results

91 in part from directed transport of autophagic proteins to presynaptic sites and their

92 local trafficking at the synapse ${ }^{7,11,12,19-21 .}$

93 Trafficking of autophagic proteins at synapses is mechanistically coupled to

94 resident synaptic proteins ${ }^{6,22-24}$. In both vertebrate and invertebrates, synaptic

95 proteins endophilin and synaptojanin 1, best known for their roles in regulating the

96 synaptic vesicle cycle, also play critical roles in the trafficking of autophagy proteins

97 and the induction of synaptic autophagy ${ }^{10,21,25,26}$. Bassoon, a large active zone

98 protein associated with synaptic vesicle retention, was similarly implicated in the

99 regulation of autophagy in neurons by interacting with the autophagy protein ATG-

$100 \quad 5^{27}$, and the E3 ubiquitin ligase Parkin ${ }^{28,29}$. These mechanistic findings support a

101 model by which the synaptic machinery, including structural active zone proteins,

102 coordinate local synaptic autophagosome biogenesis through the local regulation

103 of proteins involved in autophagy. Yet, how structural components of the synapses,

104 such as active zone proteins, regulate autophagy at synapses is not well

105 understood. 
ATG-9 is the only transmembrane protein in the core autophagy pathway ${ }^{30-}$

$103^{34}$, and is actively trafficked to promote local autophagosome biogenesis ${ }^{35-39}$,

108 including that at synapses ${ }^{12}$. ATG-9 is transported to synapses in Golgi-derived

109 vesicles, and ATG-9-containing vesicles undergo activity-dependent exo-

110 endocytosis at synapses ${ }^{21}$. Disruption of ATG-9 trafficking at synapses, including

111 its capacity to undergo exo-endocytosis, suppresses activity-dependent increases

112 in autophagy at presynaptic sites ${ }^{12,21}$. Therefore, ATG-9 trafficking at synapses is

113 important for local autophagosome biogenesis.

114 To better understand the mechanisms that regulate ATG-9 trafficking at

115 synapses, and local autophagy, we performed unbiased forward genetic screens

116 in vivo in Caenorhabditis elegans (C. elegans) neurons. We find that the long

117 isoform of the active zone protein Clarinet (CLA-1L), which bears functional

118 similarity to Piccolo and Bassoon ${ }^{40-42}$, regulates ATG-9 trafficking at synapses, and

119 presynaptic autophagy. In cla-1(L) mutants, ATG-9 abnormally accumulates into

120 clathrin-rich presynaptic structures, which can be suppressed by mutants for

121 synaptic vesicle exocytosis, suggesting that the ATG-9 phenotype in cla-1(L)

122 mutants emerges from defects in ATG-9 sorting during exo-endocytosis. We

123 observe that CLA-1L extends from the exocytic active zone to the endocytic

124 periactive zone, and genetically interacts with the periactive zone proteins EHS-

125 1/EPS15 and ITSN-1/intersectin 1 in mediating ATG-9 sorting at presynaptic sites.

126 We also find that mutants of the clathrin-associated adaptor complexes AP-2 and

127 AP180 phenocopy and enhance the ATG-9 phenotypes observed for cla-1(L)

128 mutant, whereas mutants for the AP-1 adaptor complex and the F-BAR protein 
129 syndapin 1 suppress the phenotype. Disruptions of CLA-1L that result in abnormal

130 ATG-9 localization also affect activity-dependent presynaptic autophagy.

131 Our findings support a model whereby CLA-1L bridges the exocytic active

132 zone regions with the endocytic periactive zone to regulate presynaptic sorting of

133 ATG-9, likely via endosome-mediated endocytosis. Our findings also underscore

134 the importance of active zone proteins in regulating local trafficking of autophagy

135 proteins and presynaptic autophagy, which could enable coupling of neuronal

136 activity states with biogenesis of local synaptic autophagosomes.

\section{Results}

139 The active zone protein Clarinet (CLA-1) regulates ATG-9 trafficking at 140 presynaptic sites.

141 We examined the in vivo localization of ATG-9 in the AIY interneurons of $C$.

142 elegans. AIYs are a pair of bilaterally symmetric interneurons which display a 143 stereotyped distribution of presynaptic specializations along their neurites (Figure $1441 \mathrm{~A}$ and $\mathrm{C})^{43,44}$. Simultaneous visualization of ATG-9::GFP and the presynaptic 145 marker mCherry::RAB-3 indicated that ATG-9 is enriched at presynaptic sites in 146 AIY, consistent with previous studies (Figure 1 B-D) ${ }^{12,21}$.

147 To understand ATG-9 trafficking at the presynapse and its role in regulating 148 presynaptic autophagy, we performed forward genetic screens for genes 149 regulating presynaptic localization of ATG-9. From this screen, we identified an 150 allele ${ }_{2}$ ola285, which displays abnormal ATG-9 distribution at synapses. In wild151 type animals, ATG-9 is evenly distributed in the presynaptic-rich region (termed 
152 Zone 2; Figure 1E-G and M), whereas in the ola285 mutants, ATG-9 is enriched in

153 subsynaptic foci in about sixty percent of animals (Figure $1 \mathrm{H}-\mathrm{J}$ and M). To quantify

154 the expressivity of the phenotype, we calculated an index $\left(F_{\text {peak }}-F_{\text {trough }}\right) / F_{\text {trough }}$ for

155 ATG-9::GFP. The index was calculated from representative micrographs of the

156 ATG-9 phenotype in the indicated genotypes (see Methods). Our quantifications

157 of expressivity revealed a significant redistribution of ATG-9 to subsynaptic foci in

158 ola285 mutants as compared to wild-type animals (Figure $1 \mathrm{~L}$ ).

159 To identify the genetic lesion corresponding to the ola285 allele, we

160 performed single-nucleotide polymorphism (SNP) mapping coupled with whole

161 genome sequencing ${ }^{45-47}$. We identified the genetic lesion of ola285 in the locus of

162 the gene cla-1, which encodes for Clarinet. Clarinet (CLA-1) is an active zone

163 protein that contains PDZ and C2 domains with similarity to vertebrate active zone

164 proteins Piccolo and RIM (Figure $1 \mathrm{~K})^{40-42}$. Three lines of evidence support that

165 ola285 is an allele of cla-1. First, ola285 contains a missense mutation in the cla-

1661 gene that converts Isoleucine (I) to Asparagine (N) at the residue 5753 (I5753N)

167 (Figure 1K). Second, an independent allele of clarinet, cla-1(ok560), phenocopied

168 the ATG-9 localization defects observed for ola285 mutants, both in terms of

169 penetrance and expressivity (Figure $1 \mathrm{~L}$ and $\mathrm{M})$. Third, transheterozygous animals

170 carrying both alleles ola285 and cla-1(ok560) resulted in abnormal ATG-9

171 localization at synapses, similar to that seen for either ola285 or cla-1(ok560)

172 homozygous mutants (Figure 1M). The inability of cla-1(ok560) to complement the

173 newly isolated allele ola285 suggests that they correspond to genetic lesions within

174 the same gene, cla-1. Together, our data indicate that CLA-1, an active zone 
175 protein with similarity to Piccolo and RIM, is required for trafficking of the

176 autophagy protein ATG-9 at presynaptic sites.

178 Clarinet long isoform, CLA-1L, acts cell autonomously to selectively

179 regulate ATG-9 trafficking at presynaptic sites.

180 The cla-1 gene encodes three isoforms: CLA-1L (long), CLA-1M (medium)

181 and CLA-1S (short) (Figure S1A). The long isoform, which contains a repetitive

182 region predicted to be disordered (Figure $1 \mathrm{~K}$ ), is necessary for synaptic vesicle

183 clustering, whereas the shorter isoforms are required for active zone assembly ${ }^{40}$.

184 The isolated allele cla-1(ola285), as well as the examined allele cla-1(ok560), only

185 affect CLA-1L, but not CLA-1M or CLA-1S. A null allele affecting all isoforms, cla-

1861 (wy1048), did not display a more severe phenotype than the alleles affecting only

187 CLA-1L (Figure S1A-B).

188 To determine the specific requirement of CLA-1L for ATG-9 trafficking at

189 presynaptic sites in AIY, we manipulated the expression of CLA-1L in AIY using a

190 cell-specific knockout strategy ${ }^{40}$. Briefly, the CLA-1L protein is characterized by

191 having an unusually long and unique $\mathrm{N}$-terminal region. We used a strain in which

192 loxP sites were inserted, via CRISPR, to flank this unique region (Figure 1K; Figure

193 S1A). Cell-specific expression of Cre recombinase in AIY, which leads to AIY-

194 specific deletion of the CLA-1L isoform (without affecting CLA-1S and CLA-1M),

195 resulted in the ATG-9 phenotype in AIY (Figure 1P and R), which is

196 indistinguishable from that seen for the cla-1 (ok560) allele (Figure 1Q and R,

197 compare to wild type in Figure N, O and R). Together, our data indicate that the 
198 long isoform of CLA-1 (CLA-1L) regulates presynaptic trafficking of ATG-9 in a cell199 autonomous manner.

200 Different CLA-1 isoforms play distinct roles in synaptic development, vesicle 201 clustering and release ${ }^{40}$. While the cla-1 phenotypes vary slightly between 202 neurons, previous work demonstrated that the cla-1 null allele, and not the alleles 203 of the CLA-1 long isoform, displayed phenotypes on active zone composition in 204 most neurons ${ }^{40}$. Consistent with these previous findings, we did not observe 205 phenotypes for SYD-2/Liprin $\alpha$ when comparing its distribution in the presynaptic 206 regions of AIY for wild-type and the cla-1(ok560) allele that specifically affects 207 CLA-1L isoform (Figure S1 C-E). Based on the findings that 1) the cla-1 null allele, 208 but not cla-1(L), displayed phenotypes on active zone composition, and 2) the cla2091 null allele did not display a more severe ATG-9 phenotype than cla-1(L) mutants, 210 we favor the hypothesis that the impaired ATG-9 trafficking is not due to defects in 211 active zone assembly.

212 ATG-9 is a transmembrane protein transported to the presynaptic sites in 213 vesicular structures ${ }^{12,} 21$. We therefore compared the localization of ATG-9::GFP 214 with the synaptic vesicle integral membrane protein SNG-1/synaptogyrin1::BFP 215 and the synaptic vesicle associated protein mCherry::RAB-3, both in wild-type 216 animals and cla-1(ola285) mutants. In wild-type animals, we observed a strong 217 colocalization between SNG-1 and RAB-3, as expected for synaptic vesicle 218 proteins. ATG-9 also localized to presynaptic regions, largely colocalizing with

219 these synaptic vesicle markers (Figure 2A-D). Interestingly, the phenotypes for 220 synaptic vesicle proteins and ATG-9 differ in cla-1(ola285) mutants. Unlike the 
221 ATG-9::GFP foci observed in cla-1(ola285) mutants, the synaptic vesicle markers

222 SNG-1 (or RAB-3) retained their wild type phenotype in cla-1(ola285) mutants in

223 the Zone 2 region of AIY (Figure 2E-J). Our findings suggest that the long isoform

224 of the active zone protein Clarinet specifically regulates ATG-9 trafficking at the

225 presynaptic site, and that this trafficking is distinct from that of synaptic vesicle

226 proteins.

228 ATG-9 is enriched at endocytic intermediates in cla-1(L) mutants.

ATG-9 undergoes exo-endocytosis at presynaptic sites, using synaptic

230 vesicle cycling machinery ${ }^{21}$. The cla-1(L) phenotype is reminiscent of that seen for

231 synaptic vesicle endocytosis mutants such as unc-26/synaptojanin 1, in which

232 ATG-9 accumulates in purported endocytic intermediates, and abnormally

233 colocalizes with the clathrin heavy chain subunit, CHC-1 (Figure S2A-E) ${ }^{21}$. To

234 determine if, in cla-1(L) mutants, ATG-9 similarly abnormally accumulates in

235 clathrin-rich endocytic intermediates, we examined the colocalization between

236 ATG-9 and CHC-1 in wild type and cla-1(ola285) mutant animals. We observed

237 that both ATG-9 and CHC-1 abnormally localized to synaptic foci in cla-1(ola285)

238 mutants, and colocalized with each other (Figure 3A-H and Figure S2F).

239 To determine if the abnormal accumulation of ATG-9 in cla-1(L) mutants

240 results from defects in ATG-9 sorting during exo-endocytosis, we next examined

241 the necessity of synaptic vesicle exocytosis proteins in the ATG-9 phenotype of

242 cla-1(L) mutants. We first visualized ATG-9 in putative null alleles unc-

243 13(s69)/Munc13, unc-10(md1117)/RIM, unc-18(e81)/Munc18, and unc- 
244 2(e55)/CaV2a1 (voltage-gated calcium channels), all of which encode proteins

245 essential for synaptic vesicle fusion (Figure 3I) ${ }^{48-55}$. Single mutants of unc-13(s69),

246 unc-10(md1117), unc-18(e81)/Munc18 and unc-2(e55) did not disrupt ATG-9

247 localization (Figure 3L, N, P, R and Figure S2G). Double mutants of unc-

248 13(s69);cla-1(ola285), unc-10(md1117);cla-1(ola285), unc-18(e81);cla-1(ola285)

249 and unc-2(e55);cla-1(ola285) completely suppressed abnormal ATG-9 localization

250 in cla-1(L) mutants (Figure 3M, O, Q, R and Figure S2G). These results

251 demonstrate that exocytosis is necessary for abnormal ATG-9 subsynaptic

252 accumulation into clathrin-rich foci in cla-1(ola285) mutants, and support a model

253 whereby the ATG-9 phenotype in cla-1(L) mutants results from defects in ATG-9

254 sorting during ATG-9 exo-endocytosis.

255

256 CLA-1L extends to the periactive zone and genetically interacts with 257 endocytic proteins to regulate ATG-9 trafficking.

258 Presynapses have functional subdomains that coordinate distinct steps of

259 the synaptic vesicle cycle: exocytosis takes place primarily at the active zone, while

260 endocytosis takes place primarily at the periactive zone that surrounds the active

261 zone ${ }^{56-58}$. Large active zone proteins which bear functional similarity to CLA-1L,

262 such as Piccolo and Bassoon, extend across these subdomains and have been

263 implicated in coupling synaptic vesicle exo-endocytosis at presynaptic sites in the

264 sorting of synaptic components ${ }^{57,59-65}$. CLA1L is twice the size as Piccolo and

265 Bassoon, and contains largely disordered regions which could facilitate its

266 extension from the active zone to the neighboring periactive zone. Consistent with 
267 this, in previous studies we documented that while the C-terminus of CLA-1

268 localized specifically to the active zone, the unique N-terminus of CLA-1L isoform

269 localized beyond the active zone subdomain ${ }^{40}$.

270 To better examine the relationship between CLA-1L and the presynaptic

271 subdomains, we compared the endogenous C-terminally tagged CLA-1::GFP, or

272 the endogenous N-terminally tagged GFP::CLA-1L (Figure S3A-B) ${ }^{40}$, with a 273 periactive zone marker APT-4/APA-2/AP-2 $\alpha^{66}$. While the C-terminally tagged CLA-

$274 \quad 1::$ GFP specifically localizes to small puncta corresponding to the active zone, the

275 N-terminally tagged GFP::CLA-1L displays a more distributed presynaptic pattern,

276 extending to other regions of the synaptic bouton beyond the active zone (Figure

277 4A, D, H and K). Importantly, we observed that the N-terminally tagged GFP::CLA-

$2781 \mathrm{~L}$, but not the C-terminally tagged CLA-1::GFP, colocalizes with the endocytic

279 marker APT-4/APA-2/AP-2 $\alpha$ (Figure 4A-N), which localizes to the periactive zones.

280 These findings suggest that the long isoform of CLA-1 is anchored, via its C-

281 terminus, to the active zone, but extends to the periactive zone where endocytosis

282 occurs.

283 Based on the localization of CLA-1L to these presynaptic subdomains, and

284 the ATG-9 phenotypes observed in cla-1(L) and endocytic mutants, we

285 hypothesized the existence of genetic interactions between CLA-1L and endocytic

286 proteins at the periactive zone. To test this hypothesis, we examined the

287 localization of ATG-9 in the double mutants of cla-1(ola285) with genes encoding

288 periactive zone endocytic proteins, ehs-1(ok146)/EPS15 or itsn-

289 1(ok268)/intersectin 1. We focused our analyses on the endocytic regulators EHS- 
290 1/EPS15 and ITSN-1/intersectin 1 because of their hypothesized roles in coupling

291 synaptic vesicle exocytosis at the active zone, and endocytosis at the periactive

292 zone $56,57,67-74$. We observed that in null alleles of ehs-1(ok146)/EPS15 and itsn-

293 1(ok268)/intersectin 1, thirty percent of worms displayed abnormal ATG-9 foci

294 (compared to sixty percent in cla-1(L) mutants) (Figure 4Q, S and Figure S3C).

295 Interestingly, we observed that ehs-1(ok146);cla-1(ola285) and itsn-1(ok268);cla-

296 1(ola285) enhanced the ATG-9 phenotype as compared to any of the single

297 mutants (Figure 4O-T), both in terms of penetrance (Figure S3C) and expressivity

298 (Figure 4U). Our findings uncover a cooperative genetic relationship between CLA-

$2991 \mathrm{~L}$ and the EHS-1-ITSN-1 endocytic scaffolding complex, suggesting that the

300 active zone protein CLA-1L acts in pathways that are partially redundant to the

301 EHS-1-ITSN-1 complex in linking the active zone and periactive zone regions to

302 regulate ATG-9 trafficking at presynapses.

303

304 The clathrin adaptor complexes, AP-2 and AP180, regulate ATG-9 trafficking

305 at presynaptic sites.

306 Endocytosis and sorting of transmembrane proteins depend on adaptor

307 protein complexes such as AP-2 ${ }^{75-77}$. Given our observations that in cla-1(ola285)

308 mutants, ATG-9 abnormally accumulates in clathrin-rich endocytic intermediates,

309 and that the $\mathrm{N}$-terminal region of CLA-1L co-localizes with AP-2 in the periactive

310 zone, we next examined the genetic relationship between clathrin adaptor protein

311 complexes and CLA-1L in ATG-9 localization. The AP-2 complex mediates

312 clathrin-mediated endocytosis (CME) of synaptic vesicle proteins ${ }^{78-83}$, and it has 
313 been implicated in the sorting of ATG-9 during autophagy induction in mammalian

314 non-neuronal cells ${ }^{84-86}$. To determine if the AP-2, and the associated AP180,

315 adaptor complexes were required in presynaptic trafficking of ATG-9, we examined

316 ATG-9 localization in the null alleles $d p y-23(e 840) / A P 2 \mu$ and unc-11(e47)/AP180.

317 We observed that $d p y-23(e 840) / A P 2 \mu$ and unc-11(e47)/AP180 mutants

318 phenocopied cla-1(ola285) mutants in ATG-9 presynaptic trafficking defects

319 (Figure 5A, B, C, E and F). In addition, the expressivity of the ATG-9 trafficking

320 defects was enhanced in $d p y-23(e 840) / A P 2 \mu ; c l a-1(o l a 285)$ double mutant worms

321 (Figure 5D-F). These findings suggest shared mechanisms that similarly result in

322 defective ATG-9 trafficking when clathrin-associated adaptor complexes, or the

323 active zone protein CLA-1L, are disrupted.

325 ATG-9 is sorted to endocytic intermediates via SDPN-1/syndapin 1 and the

326 AP-1 adaptor complex.

327 Clathrin-associated adaptor complexes mediate internalization and sorting

328 of cargoes from both the plasma membrane and intracellular organelles ${ }^{87,88}$. Given

329 the distinct phenotypes observed for synaptic vesicle proteins and ATG-9 upon

330 disruption of CLA-1L (Figure 2), we next investigated pathways associated with

331 clathrin-dependent endosomal sorting, the disruption of which could lead to the

332 abnormal accumulation of ATG-9 in endocytic intermediates at presynaptic sites.

333 We first examined SDPN-1/syndapin 1, a protein known to play important

334 roles in early stages of membrane invagination during both activity-dependent bulk

335 endocytosis $(\mathrm{ADBE})^{89,90}$ and ultrafast endocytosis ${ }^{91}$. We reasoned that if ATG-9 
336 was sorted via SDPN-1-dependent mechanisms, then sdpn-1 mutants would

337 suppress the observed ATG-9 foci for cla-1(L) mutants and mutants for the

338 clathrin-associated adaptor complexes. We observed that while ATG-9 localization

339 was not disrupted in sdpn-1(ok1667) single mutants, the abnormal ATG-9 foci

340 were suppressed in sdpn-1(ok1667);cla-1(ola285) or sdpn-1(ok1667);unc-

341 11(e47)/AP180 double mutant animals (Figure 6A-H). These findings are

342 consistent with the requirement of SDNP-1 in the transport of ATG-9 to

343 intermediate endosomal structures, from which CLA-1L and the clathrin-

344 associated adaptor complexes sort.

345 Next, we examined the AP-1 adaptor complex, which acts at presynaptic

346 sites to mediate endosomal sorting of activity-dependent bulk endocytosis

347 (ADBE) ${ }^{92-94}$. To determine the requirement of the AP-1 adaptor complex in ATG-9

348 sorting at presynaptic sites, we examined ATG-9 localization in unc-

349 101(m1)/AP1 $\mu 1$ single mutant animals and in double mutants with cla-1(ola285),

$350 d p y-23(e 840) / A P 2 \mu$ and unc-11(e47)/AP180. We observed that while unc-

351 101(m1)/AP1 $\mu 1$ single mutant animals did not display detectable phenotypes in

352 ATG-9 localization (Figure 7C, I and Figure S4F), unc-101(m1)/AP1 $\mu 1$ suppressed

353 the ATG-9 phenotype in double mutant animals (Figure 7A-D, G-I and Figure S4B,

354 D, E and F).

355 To confirm this, we made double mutants of cla-1(ola285) with another

356 allele, unc-101(sy108), which also suppressed the cla-1(ola285) ATG-9 phenotype

357 (Figure S4F). Furthermore, single-cell expression of the $C$. elegans cDNA of unc358 101(m1)/AP1 $\mu 1$ in AIY in the unc-101(m1);cla-1(ola285) double mutants reverted 
359 the phenotype, indicating that AP-1 acts cell autonomously in AIY to suppress the

360 cla-1(L) phenotype (Figure 7E, I and Figure S4F). The C. elegans UNC-101/AP1 11

361 is more similar to the murine AP1 11 (Query Cover: 100\%; Percentage Identity:

$36274 \%$ ) than to the murine AP2 $\mu$ (Query Cover: 98\%; Percentage Identity: 41\%)

363 (Figure S4A). To examine the conserved role of UNC-101/AP1 1 in ATG-9

364 trafficking, we expressed murine cDNA of $A P 1 \mu 1$ or AP2 $\mu$ cell specifically in AIY

365 of the unc-101(m1);cla-1(ola285) double mutants, and examined ATG-9

366 localization. We observed that expression of murine $A P 1 \mu 1$, but not $A P 2 \mu \mathrm{cDNA}$,

367 reverted the suppression seen for unc-101(m1);cla-1(ola285) double mutant

368 animals (Figure 7F, I and Figure S4C and F). Our data indicate that the AP-1

369 complex regulates ATG-9 presynaptic trafficking cell-autonomously in AIY and that

370 this function of AP-1 is conserved between mouse and C. elegans. Importantly,

371 these findings indicate that the AP-1 complex mediates ATG-9 trafficking to the

372 subsynaptic foci in cla-1(L) mutants, in AP-2 mutants and in AP180 mutants.

373 Together, our findings are consistent with a model whereby ATG-9 is sorted to

374 endocytic intermediates via proteins like AP-1 and SDPN-1, while CLA-1L, AP-2

375 and AP180 sort ATG-9 out of those endocytic intermediates (Figure 7J and K).

376

377 Disrupted ATG-9 trafficking in cla-1(L) mutants is associated with a deficit in

378 activity-induced autophagosome formation.

379 ATG-9 is important for autophagosome biogenesis at synapses ${ }^{12}$. To

380 examine how autophagosome formation is affected in cla-1(L) mutants, we

381 measured the average number of LGG-1/Atg8/GABARAP puncta (an 
382 autophagosomal marker) in the AIY neurites ${ }^{12,15,21}$ (Figure 8A-C). Previously we

383 observed the average number of LGG-1 puncta increased in AIY when the wild-

384 type animals were cultivated at $25^{\circ} \mathrm{C}^{15}$, a condition known to increase the activity

385 state of the AIY neurons ${ }^{95}$. Worms with impaired exocytosis (in unc-13 mutants) or

386 impaired autophagy (in atg-9 mutants) failed to display increased LGG-1 synaptic

387 puncta ${ }^{15,21}$. We found that, unlike wild type animals, the average number of LGG-

3881 puncta did not increase in cla-1(L) mutants (alleles ola285 and ok560) in

389 response to cultivation temperatures that increase the activity state of the neuron

390 (Figure 8D and S5A). These findings indicate that activity-induced autophagosome

391 formation at synapses is impaired in cla-1(L) mutants.

$392 \quad$ Previously, we found that in mutants that affect early stages of autophagy

393 such as epg-9(bp320), ATG-9 accumulated into subsynaptic foci, which

394 colocalized with the clathrin heavy chain $\mathrm{CHC}-1^{21}$. To determine a potential cross-

395 talk between CLA-1L-mediated ATG-9 endocytosis and autophagy, we generated

396 epg-9(bp320);cla-1(ola285) double mutant animals. Both the penetrance and the

397 expressivity of the ATG-9 phenotype are enhanced in the double mutants,

398 compared to single mutants (Figure 8E-I and S5B). The observed enhancement

399 suggests the existence of parallel pathways that sort ATG-9 at the synapse during

400 exo-endocytosis and that lead to autophagosome formation (Figure 8J).

401 Importantly, our data uncover a relationship between ATG-9 trafficking at the 402 synapse, and the activity-dependent regulation of synaptic autophagy. Together, 403 our findings are consistent with a model whereby disrupted ATG-9 trafficking in 
404 cla-1(L) mutants contributes to deficits in activity-induced autophagosome

405 formation at synapses.

406

407 Discussion

408 The long isoform of the active zone protein Clarinet (CLA-1L), regulates

409 ATG-9 trafficking at synapses and presynaptic autophagy. Autophagy, a

410 conserved cellular degradative pathway, is temporally and spatially regulated in

411 neurons to occur at synaptic compartments and in response to increased synaptic

412 activity states $6,8,10-15,18,24$. How synaptic autophagy and synaptic activity states

413 are coordinated in neurons is not well understood. From unbiased forward genetic

414 screens, we uncover a role for the active zone protein Clarinet in activity-

415 dependent autophagosome formation at synapses, and in regulated synaptic

416 trafficking of the autophagy protein, ATG-9. Our findings are consistent with recent

417 studies in primary hippocampal neurons which demonstrated that active zone

418 proteins such as Bassoon and Piccolo play important roles in synaptic protein

419 homeostasis $^{96}$, in part via the regulation of presynaptic autophagy ${ }^{27-29}$. Bassoon

420 negatively regulates presynaptic autophagy by serving as a scaffold for Atg5 ${ }^{27}$,

421 and the E3 ubiquitin ligase Parkin ${ }^{28,29}$. Our studies extend these findings in vivo in

422 C. elegans, providing further evidence for the specific roles of active zone proteins

423 in the temporal and spatial regulation of activity-dependent synaptic autophagy.

424 However, the mechanisms of CLA-1L regulation of synaptic autophagy are distinct

425 from those observed for Bassoon. We find that instead of inhibiting autophagy, 
426 CLA-1L is required for activity-dependent synaptic autophagy, likely via the

427 regulated trafficking of ATG-9 at synapses.

428 CLA-1L regulates presynaptic sorting of ATG-9 during exo-endocytosis,

429 and genetically interacts with the AP-1 and AP-2 adaptor complexes in mediating

430 ATG-9 trafficking at presynaptic sites. ATG-9 is the only transmembrane protein in

431 the core autophagy pathway, and plays key roles as a lipid scramblase to nucleate

432 the growth of the autophagosome isolation membrane ${ }^{97-100}$. In both yeast and

433 mammalian cells, ATG-9 trafficking is regulated, and its subcellular localization is

434 associated with the promotion of local autophagosome formation $35-39,84,101-105$.

435 Local autophagosome formation at synapses is associated with the degradation of

436 local synaptic cargo ${ }^{6,15,27,106-111}$, and ATG-9 trafficking at presynaptic sites via

437 exo-endocytosis could link synaptic activity states with local synaptic autophagy ${ }^{12}$

$438{ }^{21}$. We find that the active zone protein CLA-1L regulates ATG-9 trafficking during

439 its exo-endocytosis at presynaptic sites. In cla-1(L) mutants, ATG-9 abnormally

440 accumulates at clathrin-rich presynaptic foci, suggesting abnormal localization of

441 ATG-9 into endocytic intermediates. Furthermore, we find that CLA-1L genetically

442 interacts with clathrin-associated adaptor complexes, such as AP-1, AP-2 and

443 AP180, in mediating ATG-9 presynaptic trafficking. Our findings are consistent with

444 studies in non-neuronal cells which demonstrated that the AP-1 and AP-2

445 complexes mediate ATG-9 trafficking between the plasma membrane, the trans-

446 Golgi network (TGN), the recycling endosomes and the growing

447 autophagosomes $84,86,112,113$. Our findings are also consistent with recent studies

448 that uncovered roles for synaptojanin 1/UNC-26-mediated ATG-9 exo-endocytosis 
449 at synapses ${ }^{21}$. Our data extend these studies and suggest a model in which the

450 active zone protein CLA-1L is necessary for normal ATG-9 trafficking at synapses

451 in vivo, likely via the regulated clathrin-dependent sorting of ATG-9 during exo-

452 endocytosis.

453 CLA-1L spans from the exocytic active zone to the endocytic periactive

454 zone and regulates ATG-9 sorting. Clarinet is an active zone protein involved in

455 the localization of synaptic vesicle proteins, in active zone assembly, and in

456 neurotransmission ${ }^{40}$. These roles of CLA-1 are genetically separable. For

457 example, while null alleles of cla-1 that affect all three isoforms disrupt active zone

458 assembly and synapse number, alleles that specifically affect the CLA-1 long

459 isoform (CLA-1L) display specific defects in synaptic vesicle clustering, without

460 disrupting active zone assembly in most examined neurons (Figure $\mathrm{S} 1)^{40}$. We

461 similarly observe specific roles for the CLA-1L isoform in ATG-9 sorting, which we

462 interpret as distinct from the other roles of CLA-1 in active zone assembly for two

463 reasons. First, the cla-1 null allele did not display a more severe ATG-9 phenotype

464 than cla-1(L) mutants, suggesting that the ATG-9 sorting defects are due to the

465 loss of CLA-1L. Second, we do not observe ATG-9 sorting phenotypes in mutants

466 for other active zone proteins SYD-1/mSYD1A and SYD-2/Liprin- $\alpha$, known to

467 disrupt active zone assembly (Data not shown) ${ }^{114-116}$. The PDZ and C2 domains

468 of CLA-1 have sequence homology to active zone proteins RIM, Fife and Piccolo,

469 and CLA-1 has been proposed to be functionally similar to Piccolo and Bassoon in

470 its roles at the active zone ${ }^{40}$. In vertebrate synapses, Piccolo and Bassoon extend

471 from the active zone region to periactive zones, and this architecture has been 
472 proposed to be important in their purported functional roles coupling exocytosis at

473 the active zone, and protein sorting during endocytosis at the periactive zone ${ }^{57,59-}$

474 65. CLA-1L is an 8922 amino-acid protein, twice the size of Bassoon (3942 amino

475 acids) and Piccolo (4969 amino acids). It is anchored to the active zone via its C-

476 terminus ${ }^{40}$, with a large disordered $\mathrm{N}$-terminal domain extending to the periactive

477 zone, where endocytic processes occur. Consistent with these observations on

478 CLA-1L size and position at the synapses, we observe that CLA-1L regulates ATG-

4799 trafficking by genetically interacting with the periactive zone proteins EHS-

480 1/EPS15 or ITSN-1/intersectin 1, which have been suggested as linkers between

481 active-zone exocytosis and periactive zone endocytosis ${ }^{56,57,67-74}$. Our observation

482 that the ehs-1(ok146) null allele ${ }^{117}$ and itsn-1(ok268) null allele ${ }^{118}$ display

483 enhanced defects in ATG-9 sorting in cla-1L (ola285) mutants is consistent with a

484 model of functional redundancy between these proteins and CLA-1L in ATG-9

485 sorting. We propose that the specific requirement of CLA-1L for sorting of ATG-9

486 at synapses is mediated via its capacity to extend across presynaptic sub-

487 domains, from the exocytic active zone to the endocytic periactive zone.

ATG-9 likely undergoes endosome-mediated endocytosis at presynaptic

489 sites. The synaptic vesicle cycle is supported by different modes of endocytosis ${ }^{119}$,

$490{ }^{120}$, which include clathrin-mediated endocytosis $(\mathrm{CME})^{78-80,}$ 121-123, endosome-

491 mediated endocytosis (activity-dependent bulk endocytosis (ADBE) ${ }^{90,92,124-126}$ and

492 ultrafast endocytosis ${ }^{127,}{ }^{128}$. In contrast to the better-known mechanisms

493 underpinning recycling of synaptic vesicle proteins, less is known about the

494 mechanisms of ATG-9 exo-endocytosis. While we find that ATG-9 presynaptic 
495 trafficking depends on proteins such as UNC-13/Munc13 and AP-2, which also

496 regulate synaptic vesicle protein trafficking, the phenotypes of synaptic vesicle

497 proteins and ATG-9 are distinct for cla-1(L) (Figure 2), AP-2 or AP180 mutant

498 animals (data not shown). These findings indicate that although ATG-9 and

499 synaptic vesicle proteins use the same exo-endocytosis machinery, they are likely

500 trafficked and sorted via different pathways. By examining the genetic interactions

501 of adaptor protein complexes and endocytic factors in the regulation of ATG-9

502 trafficking, we propose a model whereby the AP-1 adaptor complex and the F-BAR

503 protein syndapin I (SDPN-1) mediate trafficking of ATG-9 to a transient sorting

504 station from which AP2-AP180 complexes facilitate clathrin-mediated ATG-9

505 vesicle budding (Figure 9). Consistent with observations in yeast ${ }^{36-39}$, C. elegans ${ }^{12}$

506 and non-neuronal mammalian cells ${ }^{35}$, our findings suggest that this regulated

507 ATG-9 trafficking at the synapse, and ATG-9 subcellular localization, is associated

508 with the promotion of local autophagosome formation. We hypothesize that the

509 trafficking potentiates ATG-9 role as a lipid scramblase in the nucleation of the

510 growth of the autophagosome isolation membrane ${ }^{97-100}$, perhaps via the activation

511 of the scramblase activity or ATG-9-potentiated transport of lipids necessary for

512 autophagosome biogenesis ${ }^{103,}{ }^{129}$. Our observations that mutations in early

513 autophagy protein EPG-9 similarly result in abnormal accumulation of ATG-9 in

514 synaptic foci, which is enhanced by cla-1(L) mutants, underscore the relationship

515 between ATG-9 exo-endocytosis at the synapse, active and periactive zone

516 interaction, and autophagy. 
ATG-9 trafficking at presynaptic sites via exo-endocytosis could link

518 synaptic activity with local synaptic autophagy ${ }^{21}$, thereby modulating local

519 autophagosome formation and degradation of synaptic proteins or organelles.

520 Damaged synaptic proteins and vesicles associated with synaptic vesicle recycling

521 can be degraded via autophagy $7,110,111,130$, and ATG-9 trafficking has been

522 associated with autophagosome biogenesis. Our observations are consistent with

523 a model whereby the activity dependent trafficking of ATG-9 at the synapse via

524 exo-endocytosis could track the activity state of the synapse, and instruct local

525 autophagy to degrade damaged proteins or organelles in active synapses. Active

526 zone proteins, like CLA-1L, which bridge the exocytic active zone regions with the

527 endocytic periactive zone, could regulate ATG-9 trafficking to modulate this

528 activity-dependent presynaptic autophagy.

$531 \quad$ Materials and methods

532 C. elegans strains and genetics

533 C. elegans Bristol strain worms were raised on NGM plates at $20^{\circ} \mathrm{C}$ using OP50

534 Escherichia coli as a food source ${ }^{131}$. Larva 4 (L4) stage hermaphrodites were 535 examined. For a full list of strains used in the study, please see the KEY 536 RESOURCES TABLE. 
539 Expression clones were made in the pSM vector ${ }^{132}$. The transgenic strains $(0.5-$

$54050 \mathrm{ng} / \mu \mathrm{l})$ were generated using standard injection techniques and coinjected with

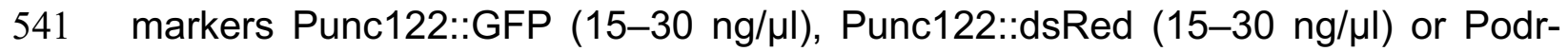

542 1::rfp (15-30ng/ $\mu \mathrm{l})$. UNC-101, mouse AP1 mu1 and mouse AP2 mu isoform1 were

543 PCR amplified from $C$. elegans and mouse cDNA (PolyATtract ${ }^{\circledR}$ mRNA Isolation

544 Systems, Promega and ProtoScript ${ }^{\circledR}$ First Strand cDNA Synthesis Kit, NEB).

545 Detailed sub-cloning information is available upon request.

547 Forward genetic screen, SNP mapping and whole-genome sequencing 548 (WGS)

549 Cla-1(ola285) was isolated from a visual forward genetic screen designed to 550 identify mutants with abnormal localization of ATG-9::GFP in the interneuron AIY.

551 Olals34 animals, expressing ATG-9::GFP and mCherry::RAB-3 in AIY

552 interneurons, were mutagenized with ethyl methanesulfonate (EMS) as described

553 previously ${ }^{131}$ and their F2 progenies were visually screened under A Leica DM

5545000 B compound microscope with an oil objective of HCX PL APO 63x/1.40-0.60

555 for abnormal ATG-9::GFP localization. Single-nucleotide polymorphism (SNP)

556 mapping ${ }^{45}$ was used to map the lesion corresponding to the ola285 allele to a 6.6-

$55711.3 \mathrm{Mbp}$ region on chromosome IV. Whole-genome sequencing (WGS) was

558 performed at the Yale Center for Genome Analysis (YCGA) and analyzed on 559 www.usegalaxy.org using "Cloudmap Unmapped Mutant workflow (w/ subtraction

560 of other strains)" as described ${ }^{46,47}$. The ola285 allele was sequenced by using

561 Sanger sequencing (Genewiz), and the genetic lesion confirmed as a single T-to- 
562 A nucleotide substitution in Exon 15 of cla-1L that results in a nonsense mutation

563 I5753N. Complementation tests were performed by generating ola285/cla-

564 1(ok560) trans-heterozygotes. The ola285 allele failed to complement cla565 1(ok560).

567 Cell autonomy of CLA-1L

568 A CRISPR protocol ${ }^{133,134}$ was used to create cla-1 (ola324), in which two loxP sites

569 were inserted into two introns of cla-1L ${ }^{40}$. Cell-specific removal of CLA-1L in AIY

570 was achieved with a plasmid driving the expression of Cre cDNA under the AIY-

571 specific mod-1 promoter fragment ${ }^{135}$.

572

\section{Cell autonomy and cell-specific rescues}

574 The ATG-9 phenotype in unc-101(m1);cla-1(ola285) was suppressed by cell 575 specifically expressing the $C$. elegans cDNA of unc-101(m1)/AP1 $1 \mathrm{C}$, and the

576 murine cDNA of $A P 1 \mu 1$. Cell-specific expression was achieved using AIY-specific

$577 \mathrm{ttx}-3 \mathrm{G}$ promoter fragment ${ }^{136}$. Cell-specific expression of the murine cDNA of $A P 2 \mu$

578 in AIY did not suppress the ATG-9 phenotype in unc-101(m1); cla-1(ola285).

580 Confocal imaging

581 Hermaphrodite animals of Larval stage 4 (L4) were anesthetized using $10 \mathrm{mM}$ 582 levamisole (Sigma-Aldrich) or 50mM muscimol in M9 buffer, mounted on 2-5\% 583 agar pads and imaged by using a 60x CFI Plan Apochromat VC, NA 1.4, oil 584 objective (Nikon) on an UltraView VoX spinning-disc confocal microscope 
585 (PerkinElmer). Images were processed with Volocity software. Maximum-intensity

586 projections presented in the figures were generated using Fiji $(\mathrm{NIH})$ for all the

587 confocal images. Quantifications were performed on maximal projections of raw

588 data.

590 Quantification and statistical analyses

591 Quantifications of penetrance and expressivity

592 A Leica DM500B compound fluorescent microscope was used to visualize and

593 screen the worms in the indicated genetic backgrounds. To quantify the

594 penetrance, animals were scored as displaying either wild type (distributed

595 throughout the Zone 2 synaptic region) or mutant (localized into subsynaptic foci)

596 phenotypes for ATG-9 or SNG-1 at Zone 2 of AIY. Mutant phenotype was defined

597 as one or more subsynaptic foci of ATG-9::GFP or SNG-1::GFP at Zone 2 of AIY.

598 For each genotype, at least 40 animals were scored. The Y-axis of graphs is

599 named as "\% of animals with ATG-9 (or SNG-1) puncta at Zone 2 of AIY". Statistics

600 for penetrance quantification was determined using two-tailed Fisher's exact test.

601 Error bars represent $95 \%$ of confidence intervals.

602 To quantify the expressivity of the phenotypes, confocal micrographs of

603 around 15 representative worms for each genotype were acquired using a

604 spinning-disc confocal microscope (PerkinElmer). The Index of ATG-9 punctum at

605 Zone 2 of AlY for each genotype was consistent across different confocal settings

606 (data not shown), so ATG-9 subsynaptic foci in cla-1(ola285) were imaged with a

607 different (lower exposure) confocal setting from the wild-type control, to avoid 
608 saturating the signal. Fluorescence values for each AIY Zone 2 were obtained after

609 background subtraction by drawing a Freehand line using Fiji along the long axis

610 of Zone 2. The fluorescence peak values and trough values were acquired via the

611 Profile Plot function. Subsynaptic enrichment index was then calculated as $\left(F_{\text {peak- }}\right.$

$612 F_{\text {trough) }} / F_{\text {trough. }}$ The $Y$-axes of the graphs are named as "Index of ATG-9 (or SNG-

613 1) punctum at Zone 2 of AIY".

614

615 Mean intensity of SYD-2 at the presynaptic Zone 2 of AlY neurons

616 To measure the level of SYD-2 at presynaptic regions, we obtained the fluorescent

617 value using Fiji as indicated above. All settings for the confocal microscope and

618 camera were kept identical to compare the intensity of SYD-2 between the wild-

619 type and cla-1(ok560) mutants (this calculation was different from that made for

620 the ATG-9 index, where the $\left(F_{\text {peak }}-F_{\text {trough }}\right) / F_{\text {trough }}$ was calculated within the same

621 synapse). The same ROI was drawn for all micrographs analyzed. The mean

622 fluorescent value of SYD-2 was measured by Fiji.

623

624 Colocalization analysis

625 The Coloc2 plugin of Fiji was used to measure the Pearson correlation coefficient

626 for colocalization between ATG-9::GFP and CHC-1::BFP, or ATG-9::GFP and

627 SNG-1::BFP, both in cla-1(ola285) mutants. ROI was drawn to include the entire

628 Zone 2 in all Z-stacks.

630 Activity-dependent autophagy 
631 To measure the synaptic autophagosomes in AIY, olals35 animals expressing

632 eGFP::LGG-1 and cytoplasmic mCherry in AIY interneurons in the wild-type, cla-

633 1(ola285) and cla-1(ok560) mutant backgrounds were raised in $20^{\circ} \mathrm{C}$ and then

634 shifted to $25^{\circ} \mathrm{C}$ for 4 hours as described ${ }^{21}$ to alter the activity state of the AIY

635 interneuron ${ }^{95}$. LGG-1 puncta in the neurite of AIY was scored under a Leica DM

$63610485000 \mathrm{~B}$ compound microscope as described ${ }^{15}$.

\section{$638 \quad$ Statistical Analyses}

639 Statistical analyses were conducted with Prism 7 software and reported in the

640 figure legends. Briefly, two-tailed Fisher's exact test was used to determine 641 statistical significance of categorical data such as the penetrance of ATG-9 642 phenotype. For continuous data, such as Index of ATG-9 punctum, two-tailed 643 unpaired Student's t-test or ordinary one-way ANOVA with Tukey's post-hoc 644 analysis was used. For LGG-1 scoring, since the number of LGG-1 puncta for each

645 condition did not pass D'Agostino \& Pearson normality test, the nonparametric 646 analysis Kruskal-Wallis with Dunn's multiple comparisons test, was used. The

647 error bars represent either 95\% confidence interval or standard deviation (SD), as 648 indicated in the figure legends, along with the $p$ values.

\section{Protein sequence alignment of the AP adaptor complex $\boldsymbol{\mu}$ subunit}

651 The sequence of $C$. elegans unc-101/AP-1 $\mu 1$ (K11D2.3) was acquired from

652 WormBase (https://wormbase.org). The sequences of murine AP-1 $\mu 1$

653 (NP_031482.1) and murine AP-2 $\mu$ (NP_001289899.1) were acquired from NCBI 
654 (https://www.ncbi.nlm.nih.gov/protein/?term=). Sequence alignment of worm

655 UNC-101/AP1 $\mu 1$, mouse AP1 $\mu 1$ and mouse AP2 $\mu$ was generated using Tcoffee

656 in Jalview 2.10.5

659 KEY RESOURCES TABLE

660

\begin{tabular}{|c|c|c|}
\hline REAGENT or RESOURCE & SOURCE & IDENTIFIER \\
\hline \multicolumn{3}{|l|}{ Chemicals } \\
\hline Levamisole Hydrochloride & Sigma-Aldrich & 31742 \\
\hline Ethyl methanesulfonate (EMS) & Sigma-Aldrich & M0880 \\
\hline Muscimol & Abcam & ab120094 \\
\hline \multicolumn{3}{|l|}{ Experimental Models: Organisms/Strains } \\
\hline $\begin{array}{l}\text { C. elegans: olals34 [pttx-3::atg-9::gfp+ pttx- } \\
\text { 3::mCh::rab-3] III }\end{array}$ & 21 & DCR4755 \\
\hline C. elegans: unc-101(m1) I & $\begin{array}{l}\text { Caenorhabditis } \\
\text { Genetics Center }\end{array}$ & DR1 \\
\hline C. elegans: unc-101(m1);olals34 & 21 & DCR8064 \\
\hline C. elegans: unc-101(m1);cla-1(ola285);olals34 & This paper & DCR8065 \\
\hline $\begin{array}{l}\text { C. elegans: olaEx4911 [pttx-3::UNC-101];unc- } \\
\text { 101(m1);cla-1(ola285);olals34 }\end{array}$ & This paper & DCR8091 \\
\hline $\begin{array}{l}\text { C. elegans: olaEx5021 [pttx-3::mouse AP- } \\
1 \mu 1 \text { ];unc-101(m1);cla-1(ola285);olals34 }\end{array}$ & This paper & DCR8296 \\
\hline $\begin{array}{l}\text { C. elegans: olaEx5023 [pttx-3::mouse AP- } \\
2 \mu 1 \text { ]; unc-101(m1);cla-1(ola285);olals34 }\end{array}$ & This paper & DCR8298 \\
\hline C. elegans: unc-101(sy108) I & $\begin{array}{l}\text { Caenorhabditis } \\
\text { Genetics Center }\end{array}$ & PS529 \\
\hline C. elegans: unc-101(sy108);olals34 & This paper & DCR8210 \\
\hline $\begin{array}{l}\text { C. elegans: unc-101(sy108);cla- } \\
\text { 1(ola285);olals34 }\end{array}$ & This paper & DCR8211 \\
\hline C. elegans: $d p y-23(e 840) \mathrm{X}$ & $\begin{array}{l}\text { Caenorhabditis } \\
\text { Genetics Center }\end{array}$ & CB840 \\
\hline C. elegans: $d p y-23(e 840) ; o l a l s 34$ & 21 & DCR8633 \\
\hline $\begin{array}{l}\text { C. elegans: dpy-23(e840);cla- } \\
\text { 1(ola285);olals34 }\end{array}$ & This paper & DCR8003 \\
\hline
\end{tabular}


C. elegans: unc-101(m1);dpy-23(e840); olals34

C. elegans: unc-26(s1710) IV

C. elegans: unc-26(s1710);olals34

C. elegans: unc-11(e47) ।

C. elegans: unc-11(e47);olals34

C. elegans: unc-101(m1);unc-11(e47);olals34

C. elegans: sdpn-1(ok1667);unc-

11(e47);olals34

C. elegans: cla-1(ola285)

C. elegans: cla-1(ola285);olals34

C. elegans: unc-18(e81) $\mathrm{X}$

C. elegans: unc-18(e81);olals34

C. elegans: unc-18(e81);cla-1(ola285);olals34

C. elegans: unc-13(s69)I

C. elegans: unc-13(s69);olals34

C. elegans: unc-13(s69);cla-1(ola285);olals34

C. elegans: unc-10(md1117) $\mathrm{X}$

C. elegans: unc-10(md1117);olals34

C. elegans: unc-10(md1117);cla-

1(ola285);olals34

C. elegans: unc-2(e55)X

C. elegans: unc-2(e55);olals34

C. elegans: unc-2(e55);cla-1(ola285);olals34

C. elegans: epg-9(bp320) IV

C. elegans: epg-9(bp320);olals34

C. elegans: epg-9(bp320);cla-

1(ola285);olals34

C. elegans: olals35 [pttx-3::egfp::lgg-1;pttx-

3::mCh]
This paper

DCR8634

Caenorhabditis

Genetics Center

21

Caenorhabditis

Genetics Center

21

EG3027

DCR6121

CB47

DCR5893

This paper

DCR8205

This paper

DCR8313

This paper

DCR8019

This paper

Caenorhabditis

Genetics Center

21

DCR8014

CB81

DCR8033

DCR8034

Erik Jorgensen $\quad B C 168$

Laboratory

(University of

Utah, Salt Lake

City, Utah)

21

This paper

Caenorhabditis

Genetics Center

This paper

This paper

DCR8031

DCR8032

Caenorhabditis

Genetics Center

CB55

This paper

This paper

Caenorhabditis

Genetics Center 21

This paper

12
DCR7047

DCR8480

DCR8204

HZ1692

DCR4750
DCR8203 


C. elegans: cla-1(ok560) IV
C. elegans: cla-1(ok560);olals34
C. elegans: cla-1(ok560);olals35
C. elegans: cla-1(ola285);olals35
C. elegans: ehs-1(ok146)II
C. elegans: ehs-1(ok146);olals34
C. elegans: ehs-1(ok146);cla-
1(ola285);olals34
C. elegans: itsn-1(ok268)IV
C. elegans: itsn-1(ok268);olals34
C. elegans: itsn-1(ok268);Cla-1(ola285);olals34
C. elegans: sdpn-1(ok1667)X

C. elegans: sdpn-1(ok1667);olals34

C. elegans: sdpn-1(ok1667);cla-

1(ola285);olals34

C. elegans: cla-1(wy1048)

C. elegans: cla-1(wy1048);olals34

C. elegans: cla-1(ola324) [floxed cla-1L]

C. elegans: cla-1(ola324) [floxed cla-1L];

olals34

C. elegans: olaEx3808 [pmod-1::nCRE]; cla-

1(ola324) [floxed cla-1L]; olals34

C. elegans: olaEx4099 [pttx-3::sng-

1::bfp];olals34

C. elegans: olaEx4099;cla-1(ola285);olals34

C. elegans: olaEx4060 [pttx-3::atg-9::mCh+

pttx-3::sng-1::gfp]

C. elegans: cla-1(ola285);olaEx4060

C. elegans: olaEx4290 [pttx-3::bfp::chc-

1];olals34

C. elegans: olaEx4290;cla-1(ola285);olals34

C. elegans: cla-1(ola311)IV [GFP::CLA-1L]

C. elegans: cla-1(ola311); olaEx5236

[punc129::apt-4::mCh]

C. elegans: cla-1(wy1186)IV [C-terminal FRTstop-FRT GFP]

C. elegans: cla-1(wy1186);olaex5237 [punc-

129::apt-4::mCh+prab-3::FLPase]

C. elegans: wyEx1826 [pttx-3::gfp::syd-2]

C. elegans: cla-1(ok560);wyEx1826

\section{Recombinant DNA}

Plasmid: pttx-3::atg-9::gfp
Caenorhabditis

Genetics Center

This paper

This paper

This paper

Caenorhabditis

Genetics Center

This paper

This paper

Caenorhabditis

Genetics Center

This paper

This paper

Caenorhabditis

Genetics Center

This paper

This paper

RB778

DCR6126

DCR7296

DCR8596

NM1568

DCR8071

DCR8234

VC201

DCR8072

DCR8235

RB1460

40

This paper

40

This paper

DCR8236

DCR8237

This paper

DCR6433

This paper

DCR6894

DCR8482

21

DCR6797

This paper

21

DCR8481

DCR7140

This paper

40

This paper

DCR8774

DCR5409

DCR5409

TV23058

This paper

DCR8698

12

TV4699

This paper
TV4699 


\begin{tabular}{|c|c|c|}
\hline Plasmid: pttx-3::sng-1::bfp & 21 & DACR2739 \\
\hline Plasmid: pttx-3::mCh::rab-3 & 43 & DACR18 \\
\hline Plasmid: pttx-3::sng-1::gfp & 21 & DACR2716 \\
\hline Plasmid: pttx-3::mCh & 12 & DACR63 \\
\hline Plasmid: pttx-3::gfp::lgg-1 & 12 & DACR1321 \\
\hline Plasmid: pttx-3::bfp:::chc-1 & 21 & DACR2877 \\
\hline Plasmid: pmod-1::nCRE & This paper & DACR2185 \\
\hline Plasmid: punc-129::apt-4::mCh & & KP-JB983 \\
\hline Plasmid: prab-3::FLPase & This paper & DACR3506 \\
\hline Plasmid: pttx-3::UNC-101 & This paper & DACR3473 \\
\hline Plasmid: pttx-3::mouse AP-1 11 & This paper & DACR3533 \\
\hline Plasmid: pttx-3::mouse AP- $2 \mu 1$ & This paper & DACR3534 \\
\hline Plasmid: pttx-3::gfp::syd-2 & 12 & DACR430 \\
\hline \multicolumn{3}{|l|}{ Software and Algorithms } \\
\hline Volocity & $\begin{array}{l}\text { Improvision by } \\
\text { Perkin Elmer }\end{array}$ & $\mathrm{N} / \mathrm{A}$ \\
\hline Fiji & 138 & $\frac{\text { https://imagej.net }}{\text { /Fiii/Downloads }}$ \\
\hline Prism & $\begin{array}{l}\text { Graphpad } \\
\text { Software Inc }\end{array}$ & $\begin{array}{l}\text { https://www.grap } \\
\text { hpad.com/ }\end{array}$ \\
\hline Adobe Illustrator & Adobe & $\begin{array}{l}\text { https://www.adob } \\
\text { e.com/products/ill } \\
\text { ustrator.html }\end{array}$ \\
\hline ApE & & $\begin{array}{l}\text { https://jorgensen. } \\
\text { biology.utah.edu/ } \\
\text { wayned/ape/ }\end{array}$ \\
\hline
\end{tabular}

\begin{tabular}{lll}
\hline Other & & \\
\hline UltraView VoX spinning disc confocal & PerkinElmer & N/A \\
microscope with a 60x CFI Plan Apo VC, NA &
\end{tabular}

1.4 , oil objective on a NikonTi-E stand 
1. Liang, Y. \& Sigrist, S. Autophagy and proteostasis in the control of synapse aging and disease. Curr Opin Neurobiol 48, 113-121 (2018).

682 2. Menzies, F.M. et al. Autophagy and Neurodegeneration: Pathogenic Mechanisms and Therapeutic Opportunities. Neuron 93, 1015-1034 (2017).

6843 3. Wang, Y., Song, M. \& Song, F. Neuronal autophagy and axon degeneration. 685 Cell Mol Life Sci 75, 2389-2406 (2018).

686 4. Luningschror, P. \& Sendtner, M. Autophagy in the presynaptic 687 compartment. Curr Opin Neurobiol 51, 80-85 (2018).

688 5. Stavoe, A.K.H. \& Holzbaur, E.L.F. Axonal autophagy: Mini-review for 689 autophagy in the CNS. Neurosci Lett 697, 17-23 (2019).

690 6. Hill, S.E. \& Colon-Ramos, D.A. The Journey of the Synaptic Autophagosome: A Cell Biological Perspective. Neuron 105, 961-973 (2020).

ealth and disease. J Cell Biol 216, 1895-1906 (2017).

694 8. Soykan, T., Haucke, V. \& Kuijpers, M. Mechanism of synaptic protein turnover and its regulation by neuronal activity. Curr Opin Neurobiol 69, 76-83 (2021).

9. Bunge, M.B. Fine structure of nerve fibers and growth cones of isolated sympathetic neurons in culture. J Cell Biol 56, 713-735 (1973). 10. Soukup, S.F. et al. A LRRK2-Dependent EndophilinA Phosphoswitch Is Critical for Macroautophagy at Presynaptic Terminals. Neuron 92, 829-844 (2016). 11. Maday, S., Wallace, K.E. \& Holzbaur, E.L. Autophagosomes initiate distally and mature during transport toward the cell soma in primary neurons. $J$ Cell Biol 196, 407-417 (2012).

704 12. Stavoe, A.K.H., Hill, S.E., Hall, D.H. \& Colon-Ramos, D.A. KIF1A/UNC-104 Transports ATG-9 to Regulate Neurodevelopment and Autophagy at Synapses. Dev Cell 38, 171-185 (2016).

13. Katsumata, K. et al. Dynein- and activity-dependent retrograde transport of autophagosomes in neuronal axons. Autophagy 6, 378-385 (2010).

14. Shehata, M., Matsumura, H., Okubo-Suzuki, R., Ohkawa, N. \& Inokuchi, K. Neuronal Stimulation Induces Autophagy in Hippocampal Neurons That Is Involved in AMPA Receptor Degradation after Chemical Long-Term Depression. $J$ Neurosci 32, 10413-10422 (2012).

713 15. Hill, S.E. et al. Maturation and Clearance of Autophagosomes in Neurons

714 Depends on a Specific Cysteine Protease Isoform, ATG-4.2. Dev Cell 49, 251-+ 715 (2019).

716 16. Maday, S. \& Holzbaur, E.L. Compartment-Specific Regulation of Autophagy 717 in Primary Neurons. J Neurosci 36, 5933-5945 (2016).

718 17. Wang, T. et al. Control of autophagosome axonal retrograde flux by 719 presynaptic activity unveiled using botulinum neurotoxin type-A. J Neurochem 134, 720 165-165 (2015).

721 18. Kulkarni, V.V. et al. Synaptic activity controls autophagic vacuole motility 722 and function in dendrites. Journal of Cell Biology 220 (2021). 
723 19. Soukup, S.F. \& Verstreken, P. EndoA/Endophilin-A creates docking 724 stations for autophagic proteins at synapses. Autophagy 13, 971-972 (2017).

725 20. Maday, S. \& Holzbaur, E.L. Autophagosome biogenesis in primary neurons

726 follows an ordered and spatially regulated pathway. Dev Cell 30, 71-85 (2014).

727 21. Yang, S. et al. Presynaptic autophagy is coupled to the synaptic vesicle 728 cycle via ATG-9. bioRxiv, 2020.2012.2028.424508 (2020).

729 22. Kuijpers, M., Azarnia Tehran, D., Haucke, V. \& Soykan, T. The axonal 730 endolysosomal and autophagic systems. J Neurochem (2020).

731 23. Andres-Alonso, M., Kreutz, M.R. \& Karpova, A. Autophagy and the endolysosomal system in presynaptic function. Cell Mol Life Sci 78, 2621-2639 (2021).

24. Stavoe, A.K.H. \& Holzbaur, E.L.F. Autophagy in Neurons. Annu Rev Cell Dev Bi 35, 477-500 (2019).

25. George, A.A., Hayden, S., Stanton, G.R. \& Brockerhoff, S.E. Arf6 and the 5 'phosphatase of synaptojanin 1 regulate autophagy in cone photoreceptors. Bioessays 38 Suppl 1, S119-135 (2016).

26. Vanhauwaert, R. et al. The SAC1 domain in synaptojanin is required for autophagosome maturation at presynaptic terminals. Embo $J$ 36, 1392-1411 (2017).

27. Okerlund, N.D. et al. Bassoon Controls Presynaptic Autophagy through Atg5. Neuron 93, 897-913 e897 (2017).

28. Montenegro-Venegas, C., Annamneedi, A., Hoffmann-Conaway, S., Gundelfinger, E.D. \& Garner, C.C. BSN (bassoon) and PRKN/parkin in concert control presynaptic vesicle autophagy. Autophagy 16, 1732-1733 (2020).

29. Hoffmann-Conaway, $S$. et al. Parkin contributes to synaptic vesicle autophagy in Bassoon-deficient mice. Elife 9 (2020).

30. Noda, T. et al. Apg9p/Cvt7p is an integral membrane protein required for transport vesicle formation in the Cvt and autophagy pathways. J Cell Biol 148, 465-480 (2000).

31. Noda, T. Autophagy in the context of the cellular membrane-trafficking system: the enigma of Atg9 vesicles. Biochem Soc T 45, 1323-1331 (2017).

32. Webber, J.L. \& Tooze, S.A. New insights into the function of Atg9. Febs Letters 584, 1319-1326 (2010).

33. Guardia, C.M. et al. The structure of human ATG9A and its interplay with the lipid bilayer. Autophagy, 1-2 (2020).

34. Lang, T., Reiche, S., Straub, M., Bredschneider, M. \& Thumm, M. Autophagy and the cvt pathway both depend on AUT9. J Bacteriol 182, 2125-2133 (2000).

35. Karanasios, E. et al. Autophagy initiation by ULK complex assembly on ER tubulovesicular regions marked by ATG9 vesicles. Nature Communications 7 (2016).

766

36. Reggiori, F., Tucker, K.A., Stromhaug, P.E. \& Klionsky, D.J. The Atg1-Atg13 complex regulates Atg9 and Atg23 retrieval transport from the preautophagosomal structure. Dev Cell 6, 79-90 (2004). 
767 37. Reggiori, F., Shintani, T., Nair, U. \& Klionsky, D.J. Atg9 cycles between 768 mitochondria and the pre-autophagosomal structure in yeasts. Autophagy 1, 101769109 (2005).

770 38. Sawa-Makarska, J. et al. Reconstitution of autophagosome nucleation defines Atg9 vesicles as seeds for membrane formation. Science 369 (2020).

39. Sekito, T., Kawamata, T., Ichikawa, R., Suzuki, K. \& Ohsumi, Y. Atg17 recruits Atg9 to organize the pre-autophagosomal structure. Genes Cells 14, 525538 (2009).

40. Xuan, Z. et al. Clarinet (CLA-1), a novel active zone protein required for synaptic vesicle clustering and release. Elife 6 (2017).

41. Mullen, G. et al. The C. elegans RIM-Piccolo homolog CLA-1 is required for normal synaptic transmission. International Worm Meeting (2017). WormBase ID: WBPaper00052816 (2017).

42. Rand, J. et al. The rip-1 gene encodes a set of presynaptic proteins similar to vertebrate RIM and Piccolo. International Worm Meeting (2003). WormBase ID: WBPaper00019364 (2003)

43. Colon-Ramos, D.A., Margeta, M.A. \& Shen, K. Glia promote local synaptogenesis through UNC-6 (netrin) signaling in C-elegans. Science 318, 103106 (2007).

44. White, J.G., Southgate, E., Thomson, J.N. \& Brenner, S. The structure of the nervous system of the nematode Caenorhabditis elegans. Philos Trans $R$ Soc Lond B Biol Sci 314, 1-340 (1986).

45. Davis, M.W. et al. Rapid single nucleotide polymorphism mapping in Celegans. Bmc Genomics 6 (2005).

46. Doitsidou, M., Poole, R.J., Sarin, S., Bigelow, H. \& Hobert, O. C. elegans Mutant Identification with a One-Step Whole-Genome-Sequencing and SNP Mapping Strategy. Plos One 5 (2010).

47. Minevich, G., Park, D.S., Blankenberg, D., Poole, R.J. \& Hobert, O. CloudMap: a cloud-based pipeline for analysis of mutant genome sequences. Genetics 192, 1249-1269 (2012).

48. Richmond, J.E., Davis, W.S. \& Jorgensen, E.M. UNC-13 is required for synaptic vesicle fusion in C-elegans. Nat Neurosci 2, 959-964 (1999).

49. Koushika, S.P. et al. A post-docking role for active zone protein Rim. Nat Neurosci 4, 997-1005 (2001).

50. Hata, Y., Slaughter, C.A. \& Sudhof, T.C. Synaptic vesicle fusion complex contains unc-18 homologue bound to syntaxin. Nature 366, 347-351 (1993).

51. Sassa, T. et al. Regulation of the UNC-18-Caenorhabditis elegans syntaxin complex by UNC-13. J Neurosci 19, 4772-4777 (1999).

52. Schafer, W.R. \& Kenyon, C.J. A Calcium-Channel Homolog Required for Adaptation to Dopamine and Serotonin in Caenorhabditis-Elegans. Nature 375, 73-78 (1995).

53. Gracheva, E.O., Hadwiger, G., Nonet, M.L. \& Richmond, J.E. Direct interactions between C. elegans RAB-3 and Rim provide a mechanism to target vesicles to the presynaptic density. Neuroscience Letters 444, 137-142 (2008). 
811 54. Han, Y., Kaeser, P.S., Sudhof, T.C. \& Schneggenburger, R. RIM 812 determines $\mathrm{Ca}(2)+$ channel density and vesicle docking at the presynaptic active 813 zone. Neuron 69, 304-316 (2011).

814 55. Kaeser, P.S. et al. RIM Proteins Tether Ca2+ Channels to Presynaptic 815 Active Zones via a Direct PDZ-Domain Interaction. Cell 144, 282-295 (2011).

816 56. Haucke, V., Neher, E. \& Sigrist, S.J. Protein scaffolds in the coupling of 817 synaptic exocytosis and endocytosis. Nat Rev Neurosci 12, 127-138 (2011).

818 57. Maritzen, T. \& Haucke, V. Coupling of exocytosis and endocytosis at the 819 presynaptic active zone. Neurosci Res 127, 45-52 (2018).

820 58. Lou, X.L. Sensing Exocytosis and Triggering Endocytosis at Synapses: 821 Synaptic Vesicle Exocytosis-Endocytosis Coupling. Frontiers in Cellular 822 Neuroscience 12 (2018).

823 59. Mukherjee, K. et al. Piccolo and bassoon maintain synaptic vesicle 824 clustering without directly participating in vesicle exocytosis. P Natl Acad Sci USA 825 107, 6504-6509 (2010).

826 60. Fenster, S.D. et al. Piccolo, a presynaptic zinc finger protein structurally 827 related to bassoon. Neuron 25, 203-214 (2000).

828 61. Gundelfinger, E.D., Reissner, C. \& Garner, C.C. Role of Bassoon and 829 Piccolo in Assembly and Molecular Organization of the Active Zone. Front Synaptic 830 Neurosci 7, 19 (2015).

831 62. Dieck, S.T. et al. Bassoon, a novel zinc-finger CAG/glutamine-repeat 832 protein selectively localized at the active zone of presynaptic nerve terminals. 833 Journal of Cell Biology 142, 499-509 (1998).

834 63. CasesLanghoff, C. et al. Piccolo, a novel $420 \mathrm{kDa}$ protein associated with 835 the presynaptic cytomatrix. Eur J Cell Biol 69, 214-223 (1996).

836 64. Dani, A., Huang, B., Bergan, J., Dulac, C. \& Zhuang, X.W. Superresolution 837 Imaging of Chemical Synapses in the Brain. Neuron 68, 843-856 (2010).

838 65. Fenster, S.D. et al. Interactions between Piccolo and the actin/dynamin839 binding protein Abp1 link vesicle endocytosis to presynaptic active zones. J Biol 840 Chem 278, 20268-20277 (2003).

841 66. Bai, J.H., Hu, Z.T., Dittman, J.S., Pym, E.C.G. \& Kaplan, J.M. Endophilin 842 Functions as a Membrane-Bending Molecule and Is Delivered to Endocytic Zones 843 by Exocytosis. Cell 143, 430-441 (2010).

844 67. Koh, T.W. et al. Eps15 and Dap160 control synaptic vesicle membrane 845 retrieval and synapse development. J Cell Biol 178, 309-322 (2007).

846 68. Pechstein, A. et al. Regulation of synaptic vesicle recycling by complex 847 formation between intersectin 1 and the clathrin adaptor complex AP2. P Natl Acad 848 Sci USA 107, 4206-4211 (2010).

849 69. Koh, T.W., Verstreken, P. \& Bellen, H.J. Dap160/intersectin acts as a 850 stabilizing scaffold required for synaptic development and vesicle endocytosis. 851 Neuron 43, 193-205 (2004).

852 70. Marie, B. et al. Dap160/intersectin scaffolds the periactive zone to achieve 853 high-fidelity endocytosis and normal synaptic growth. Neuron 43, 207-219 (2004).

854 71. Gubar, O. et al. Intersectin: The Crossroad between Vesicle Exocytosis and 855 Endocytosis. Front Endocrinol (Lausanne) 4, 109 (2013). 
856 72. Wang, W. et al. ITSN-1 controls vesicle recycling at the neuromuscular 857 junction and functions in parallel with DAB-1. Traffic 9, 742-754 (2008).

858 73. Gowrisankaran, S. et al. Endophilin-A coordinates priming and fusion of neurosecretory vesicles via intersectin. Nat Commun 11, 1266 (2020).

860 74. Mishra, R., Sengul, G.F., Candiello, E. \& Schu, P. Synaptic AP2 CCV life 861 cycle regulation by the Eps15, ITSN1, Sgip1/AP2, synaptojanin1 interactome. Sci 862 Rep 11, 8007 (2021).

863 75. Robinson, M.S. Adaptable adaptors for coated vesicles. Trends Cell Biol 864 14, 167-174 (2004).

865 76. Kelly, B.T. \& Owen, D.J. Endocytic sorting of transmembrane protein cargo. 866 Curr Opin Cell Biol 23, 404-412 (2011).

867 77. Guardia, C.M., De Pace, R., Mattera, R. \& Bonifacino, J.S. Neuronal 868 functions of adaptor complexes involved in protein sorting. Curr Opin Neurobiol 51, 869 103-110 (2018).

870 78. Maycox, P.R., Link, E., Reetz, A., Morris, S.A. \& Jahn, R. Clathrin-coated 871 vesicles in nervous tissue are involved primarily in synaptic vesicle recycling. $J$ Cell 872 Biol 118, 1379-1388 (1992).

873 79. Royle, S.J. \& Lagnado, L. Endocytosis at the synaptic terminal. J Physiol874 London 553, 345-355 (2003).

875 80. Takei, K., Mundigl, O., Daniell, L. \& DeCamilli, P. The synaptic vesicle cycle: 876 A single vesicle budding step involving clathrin and dynamin. Journal of Cell 877 Biology 133, 1237-1250 (1996).

878 81. Keen, J.H. \& Beck, K.A. Identification of the clathrin-binding domain of 879 assembly protein AP-2. Biochem Biophys Res Commun 158, 17-23 (1989).

880 82. Traub, L.M. Sorting it out: AP-2 and alternate clathrin adaptors in endocytic 881 cargo selection. Journal of Cell Biology 163, 203-208 (2003).

882 83. Hirst, J. \& Robinson, M.S. Clathrin and adaptors. Bba-Mol Cell Res 1404, 883 173-193 (1998).

884 84. Puri, C., Renna, M., Bento, C.F., Moreau, K. \& Rubinsztein, D.C. Diverse 885 autophagosome membrane sources coalesce in recycling endosomes. Cell 154, 886 1285-1299 (2013).

887 85. Popovic, D. \& Dikic, I. TBC1D5 and the AP2 complex regulate ATG9 888 trafficking and initiation of autophagy. EMBO Rep 15, 392-401 (2014).

889 86. Imai, K. et al. Atg9A trafficking through the recycling endosomes is required 890 for autophagosome formation. J Cell Sci 129, 3781-3791 (2016).

891 87. McMahon, H.T. \& Boucrot, E. Molecular mechanism and physiological 892 functions of clathrin-mediated endocytosis. Nat Rev Mol Cell Biol 12, 517-533 893 (2011).

894 88. Nakatsu, F. \& Ohno, H. Adaptor protein complexes as the key regulators of 895 protein sorting in the post-Golgi network. Cell Struct Funct 28, 419-429 (2003).

896 89. Cheung, G. \& Cousin, M.A. Synaptic vesicle generation from activity897 dependent bulk endosomes requires a dephosphorylation-dependent dynamin898 syndapin interaction. J Neurochem 151, 570-583 (2019).

899 90. Clayton, E.L. \& Cousin, M.A. The molecular physiology of activity900 dependent bulk endocytosis of synaptic vesicles. J Neurochem 111, 901-914 901 (2009). 
902 91. Imoto, Y. et al. Dynamin is primed at endocytic sites for ultrafast 903 endocytosis. bioRxiv, 2021.2002.2015.431332 (2021).

904 92. Cheung, G. \& Cousin, M.A. Adaptor protein complexes 1 and 3 are essential 905 for generation of synaptic vesicles from activity-dependent bulk endosomes. $J$ 906 Neurosci 32, 6014-6023 (2012).

907 93. Glyvuk, N. et al. AP-1/sigma1B-adaptin mediates endosomal synaptic 908 vesicle recycling, learning and memory. Embo J 29, 1318-1330 (2010).

909 94. Park, J., Cho, O.Y., Kim, J.A. \& Chang, S. Endosome-mediated endocytic 910 mechanism replenishes the majority of synaptic vesicles at mature CNS synapses 911 in an activity-dependent manner. Sci Rep-Uk 6 (2016).

912 95. Hawk, J.D. et al. Integration of Plasticity Mechanisms within a Single 913 Sensory Neuron of C. elegans Actuates a Memory. Neuron 97, 356-+ (2018).

914 96. Waites, C.L. et al. Bassoon and Piccolo maintain synapse integrity by 915 regulating protein ubiquitination and degradation. Embo J 32, 954-969 (2013).

916 97. Matoba, K. et al. Atg9 is a lipid scramblase that mediates autophagosomal 917 membrane expansion. Nat Struct Mol Biol (2020).

918 98. Maeda, S. et al. Structure, lipid scrambling activity and role in autophagosome formation of ATG9A. Nat Struct Mol Biol (2020).

99. Matoba, K. \& Noda, N.N. Secret of Atg9: lipid scramblase activity drives de novo autophagosome biogenesis. Cell Death Differ (2020).

100. Ghanbarpour, A., Valverde, D.P., Melia, T.J. \& Reinisch, K.M. A model for a partnership of lipid transfer proteins and scramblases in membrane expansion and organelle biogenesis. P Natl Acad Sci USA 118 (2021).

101. Mari, M. et al. An Atg9-containing compartment that functions in the early steps of autophagosome biogenesis. J Cell Biol 190, 1005-1022 (2010).

102. Suzuki, K. et al. The pre-autophagosomal structure organized by concerted functions of APG genes is essential for autophagosome formation. Embo $\mathrm{J} 20$, 5971-5981 (2001).

103. Yamamoto, $\mathrm{H}$. et al. Atg9 vesicles are an important membrane source during early steps of autophagosome formation. Journal of Cell Biology 198, 219233 (2012).

104. Webber, J.L., Young, A.R.J. \& Tooze, S.A. Atg9 trafficking in mammalian cells. Autophagy 3, 54-56 (2007).

105. Mari, M. \& Reggiori, F. Atg9 trafficking in the yeast Saccharomyces cerevisiae. Autophagy 3, 145-148 (2007).

106. Hollenbeck, P.J. Products of endocytosis and autophagy are retrieved from axons by regulated retrograde organelle transport. J Cell Biol 121, 305-315 (1993). 107. Hollenbeck, P.J. \& Bray, D. Rapidly Transported Organelles Containing Membrane and Cytoskeletal Components - Their Relation to Axonal Growth. Journal of Cell Biology 105, 2827-2835 (1987).

108. Ban, B.K. et al. Autophagy negatively regulates early axon growth in cortical neurons. Mol Cell Biol 33, 3907-3919 (2013).

109. Hernandez, D. et al. Regulation of presynaptic neurotransmission by macroautophagy. Neuron 74, 277-284 (2012).

947 pathway. Elife 4, e05597 (2015). 
948 111. Hoffmann, S. et al. Light-Activated ROS Production Induces Synaptic 949 Autophagy. J Neurosci 39, 2163-2183 (2019).

950 112. Zhou, C. et al. Regulation of mATG9 trafficking by Src- and ULK1-mediated 951 phosphorylation in basal and starvation-induced autophagy. Cell Res 27, 184-201 952 (2017).

953 113. Guo, Y.J. et al. AP1 is essential for generation of autophagosomes from the 954 trans-Golgi network. J Cell Sci 125, 1706-1715 (2012).

955 114. Hallam, S.J., Goncharov, A., McEwen, J., Baran, R. \& Jin, Y.S. SYD-1, a presynaptic protein with PDZ, C2 and rhoGAP-like domains, specifies axon identity in C-elegans. Nat Neurosci 5, 1137-1146 (2002).

115. Kittelmann, M. et al. Liprin-alpha/SYD-2 determines the size of dense projections in presynaptic active zones in C. elegans. Journal of Cell Biology 203, 849-863 (2013).

961 116. Zhen, M. \& Jin, Y. The liprin protein SYD-2 regulates the differentiation of presynaptic termini in C. elegans. Nature 401, 371-375 (1999). 117. Salcini, A.E. et al. The Eps15 C-elegans homologue EHS-1 is implicated in synaptic vesicle recycling. Nat Cell Biol 3, 755-760 (2001). 118. Rose, $\mathrm{S}$. et al. Caenorhabditis elegans intersectin: a synaptic protein regulating neurotransmission. Mol Biol Cell 18, $5091-5099$ (2007).

119. Milosevic, I. Revisiting the Role of Clathrin-Mediated Endoytosis in Synaptic Vesicle Recycling. Frontiers in Cellular Neuroscience 12 (2018).

969 120. Smith, S.M., Renden, R. \& von Gersdorff, H. Synaptic vesicle endocytosis: 970 fast and slow modes of membrane retrieval. Trends Neurosci 31, 559-568 (2008). 121. Heuser, J.E. \& Reese, T.S. Evidence for Recycling of Synaptic Vesicle Membrane during Transmitter Release at Frog Neuromuscular Junction. Journal of Cell Biology 57, 315-344 (1973).

974 122. Saheki, Y. \& De Camilli, P. Synaptic vesicle endocytosis. Cold Spring Harb Perspect Biol 4, a005645 (2012).

976 123. Pfeffer, S.R. \& Kelly, R.B. The Subpopulation of Brain Coated Vesicles That Carries Synaptic Vesicle Proteins Contains 2 Unique Polypeptides. Cell 40, 949957 (1985).

979 124. Holt, M., Cooke, A., Wu, M.M. \& Lagnado, L. Bulk membrane retrieval in the synaptic terminal of retinal bipolar cells. J Neurosci 23, 1329-1339 (2003).

125. Miller, T.M. \& Heuser, J.E. Endocytosis of Synaptic Vesicle Membrane at the Frog Neuromuscular-Junction. Journal of Cell Biology 98, 685-698 (1984).

990 126. Wu, W. \& Wu, L.G. Rapid bulk endocytosis and its kinetics of fission pore closure at a central synapse. P Natl Acad Sci USA 104, 10234-10239 (2007).

127. Watanabe, S. et al. Ultrafast endocytosis at Caenorhabditis elegans neuromuscular junctions. Elife 2 (2013).

128. Watanabe, S. et al. Ultrafast endocytosis at mouse hippocampal synapses. Nature 504, 242-+ (2013).

129. Feng, Y.C. \& Klionsky, D.J. Autophagic membrane delivery through ATG9. Cell Research 27, 161-162 (2017).

991 130. Cohen, L.D. \& Ziv, N.E. Recent insights on principles of synaptic protein 992 degradation. F1000Res 6, 675 (2017). 
993 131. Brenner, S. The genetics of Caenorhabditis elegans. Genetics 77, 71-94 994 (1974).

995 132. Shen, K. \& Bargmann, C.I. The immunoglobulin superfamily protein SYG-1

996 determines the location of specific synapses in C. elegans. Cell 112, 619-630 997 (2003).

998 133. Paix, A. et al. Scalable and Versatile Genome Editing Using Linear DNAs 999 with Microhomology to Cas9 Sites in Caenorhabditis elegans. Genetics 198, 1347$1000+(2014)$.

1001 134. Arribere, J.A. et al. Efficient Marker-Free Recovery of Custom Genetic 1002 Modifications with CRISPR/Cas9 in Caenorhabditis elegans. Genetics 198, 8371003 U842 (2014).

1004 135. Flavell, S.W. et al. Serotonin and the Neuropeptide PDF Initiate and Extend 1005 Opposing Behavioral States in C. elegans. Cell 154, 1023-1035 (2013). 136. Wenick, A.S. \& Hobert, O. Genomic cis-regulatory architecture and transacting regulators of a single interneuron-specific gene battery in C-elegans. Dev Cell 6, 757-770 (2004). 137. Waterhouse, A.M., Procter, J.B., Martin, D.M., Clamp, M. \& Barton, G.J. Jalview Version 2--a multiple sequence alignment editor and analysis workbench. Bioinformatics 25, 1189-1191 (2009).

1012

1013

138. Schindelin, J. et al. Fiji: an open-source platform for biological-image analysis. Nat Methods 9, 676-682 (2012).

1015 139. Clark, D.A., Biron, D., Sengupta, P. \& Samuel, A.D.T. The AFD sensory neurons encode multiple functions underlying thermotactic behavior in

1016 Caenorhabditis elegans. J Neurosci 26, 7444-7451 (2006).

1017 140. Biron, D. et al. A diacylglycerol kinase modulates long-term thermotactic behavioral plasticity in C. elegans. Nat Neurosci 9, 1499-1505 (2006).

\section{Acknowledgments}

1023 We thank Jihong Bai (Basic Sciences Division, Fred Hutch) and Kang Shen

1024 (Department of Biology, Stanford University) for providing strains and constructs.

1025 We thank Lin Shao (Department of Neuroscience, Yale University) for assistance

1026 with image quantification and statistics. We thank Josh Hawk for providing mouse

1027 cDNA. We thank current and past members of the Colón-Ramos lab for help,

1028 advice and insightful comments on the project. We also thank Andrea Stavoe, Mia

1029 Dawn, Peri Kurshan, Janet Richmond and Pietro De Camilli for comments on the 1030 project. We thank the Caenorhabditis Genetics Center (funded by NIH Office of 
1031 Research Infrastructure Programs P40 OD010440) for C. elegans strains. S.Y.

1032 was supported by China Scholarship Council-Yale World Scholars Program.

1033 Research in the D.A.C.-R. lab was supported by NIH R01NS076558,

1034 DP1NS111778 and by an HHMI Scholar Award.

1035

\section{Contributions}

1037 Z. X., S.Y., and D.C.R. conceived the study and designed the experiments. Z.X.,

1038 S.Y., and S.E.H. conducted the experiments. Z.X. performed image quantification

1039 and biostatistics analysis. Z.X. and D.C.R. prepared the manuscript with

1040 contribution from S.Y., S.E.H., B.C., and L.M. 


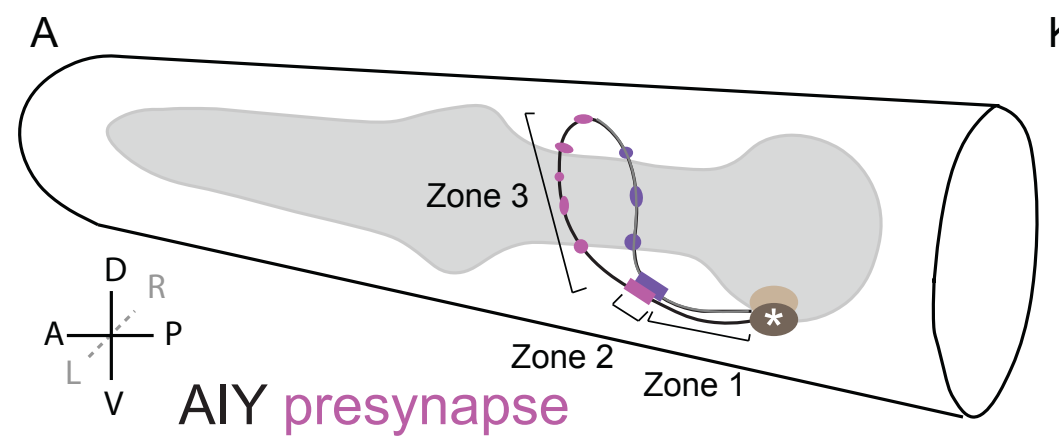

K
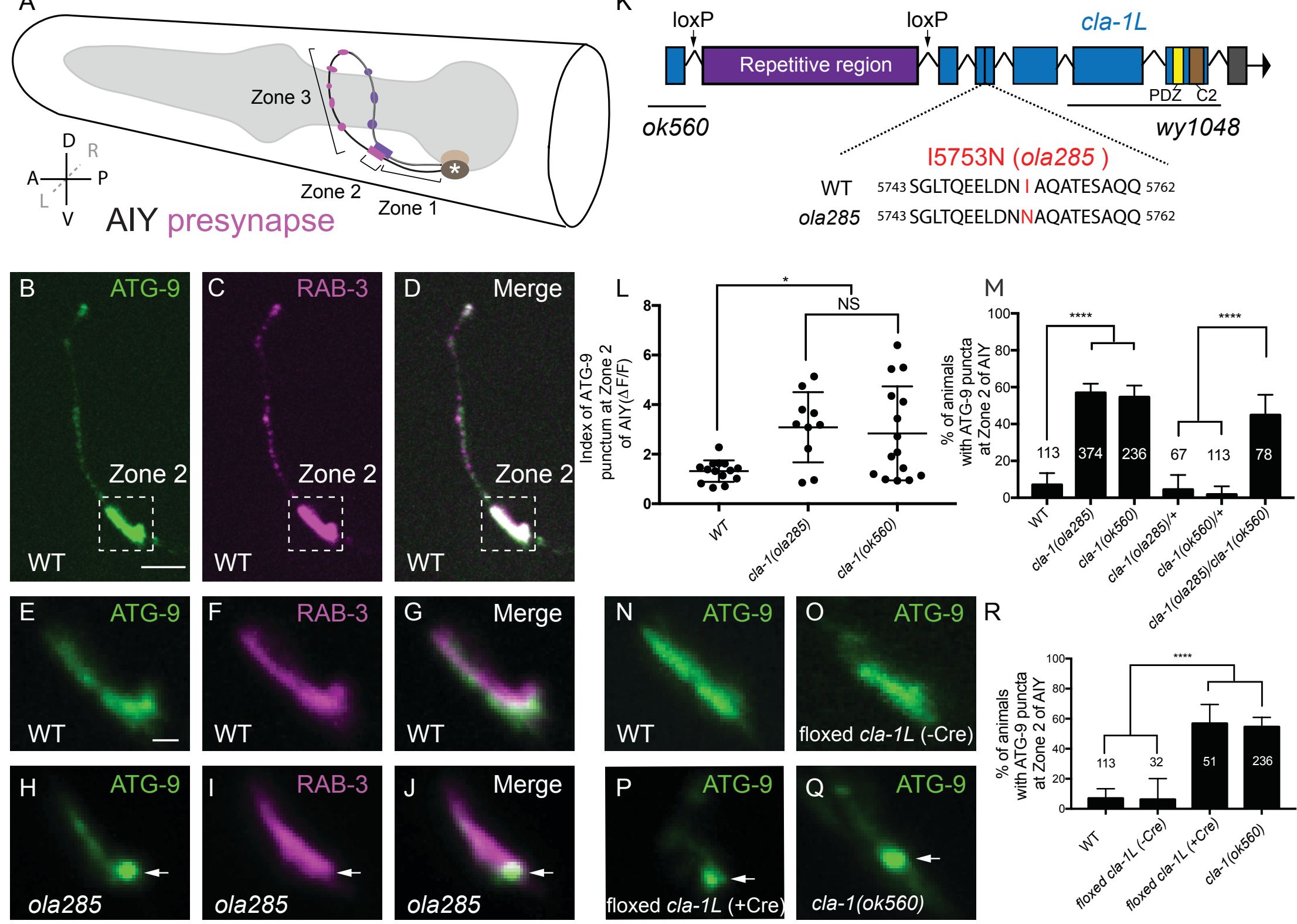

Figure 1 


\section{Figure 1. The long isoform of Clarinet (CLA-1L) regulates ATG-9 trafficking}

\section{5 at presynaptic sites.}

1056 (A) Schematic of the head of C. elegans, including pharynx (grey region) and the 1057 two bilaterally symmetric AIY interneurons. The asterisk denotes the cell body.

1058 There are three distinct segments along the AIY neurite: an asynaptic region 1059 proximal to AIY cell body (Zone 1), a large presynaptic region (Zone 2), and a 1060 segment with discrete presynaptic clusters at the distal part of the neurite (Zone $10613)^{43,44}$. Presynaptic regions (Zone 2 and Zone 3) are in magenta (AIYL) or violet 1062 (AIYR). In axis, A, anterior; P, posterior; L, left; R, right; D, dorsal; V, ventral.

1063 (B-D) Distribution of ATG-9::GFP (B) and synaptic vesicle protein (mCherry::RAB-

10643 , pseudo-colored magenta) (C) in the synaptic regions of AIY (merge in D). The 1065 dashed box encloses AIY Zone 2.

1066 (E-J) Distribution of ATG-9::GFP (E and $H$ ) and synaptic vesicle protein

1067 (mCherry::RAB-3, pseudo-colored magenta) (F and I) at Zone 2 of AIY (merge in

1068 G and J) in wild type (WT) (E-G) and ola285 mutant (H-J) animals. ATG-9 is evenly 1069 distributed in WT, but forms subsynaptic foci in ola285 mutants, which are not 1070 enriched with RAB-3 (indicated by arrows in $\mathrm{H}-\mathrm{J}$ ).

1071 (K) Schematic of the genomic region of cla-1L. The locations of loxP sites and the 1072 genetic lesions of the cla-1 alleles examined in this study are indicated. The 1073 genetic lesion in allele ola285 (I to $\mathrm{N}$ at residue 5753) is shown for both WT and 1074 ola285 mutants. The positions of the repetitive region in CLA-1L, and the 1075 conserved PDZ and C2 domains in all CLA-1 isoforms are also shown in the 1076 schematic. 
1077 (L) Quantification of the index of ATG-9 punctum ( $\Delta F / F$; See Methods) at Zone 2

1078 of AIY in wild type (WT), cla-1(ola285) and cla-1(ok560) mutants. Error bars show

1079 standard deviation (SD). "NS" (not significant), ${ }^{*} p<0.05$ by ordinary one-way

1080 ANOVA with Tukey's multiple comparisons test. Each dot in the scatter plot

1081 represents a single animal.

1082 (M) Quantification of the percentage of animals displaying ATG-9 subsynaptic foci 1083 at AIY Zone 2 in the indicated genotypes. Error bars represent 95\% confidence 1084 interval. ${ }^{* * *} p<0.0001$ by two-tailed Fisher's exact test. The number on the bars 1085 indicates the number of animals scored.

1086 (N-Q) Distribution of ATG-9::GFP at Zone 2 of AIY in wild type (WT) (N), floxed 1087 cla-1L without Cre $(\mathrm{O})$ and floxed cla-1L with Cre expressed cell-specifically in AIY 1088 (P) and cla-1(ok560) (Q) animals. Arrows (in P and Q) indicate abnormal ATG-9 1089 foci.

1090 (R) Quantification of the percentage of animals displaying ATG-9 subsynaptic foci 1091 at AIY Zone 2 in the indicated genotypes. Error bars represent 95\% confidence 1092 interval. ${ }^{* * * *} p<0.0001$ by two-tailed Fisher's exact test. The number on the bar 1093 indicates the number of animals scored.

1094 Scale bar (in B for B-D), $5 \mu \mathrm{m}$; (in E for E-J and N-Q), $1 \mu \mathrm{m}$. 

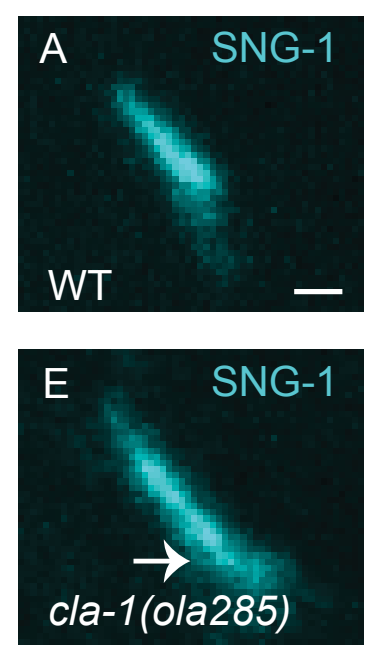

造

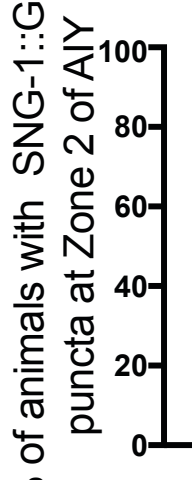
우
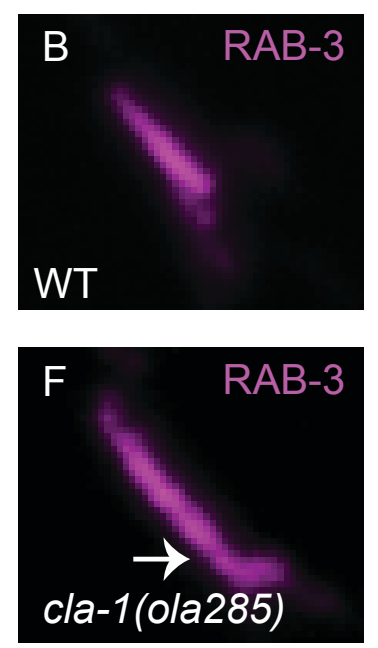
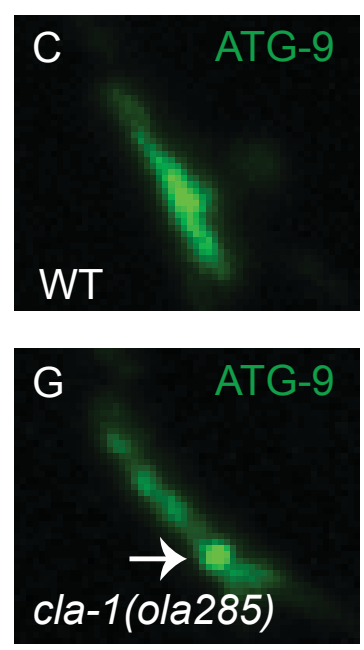
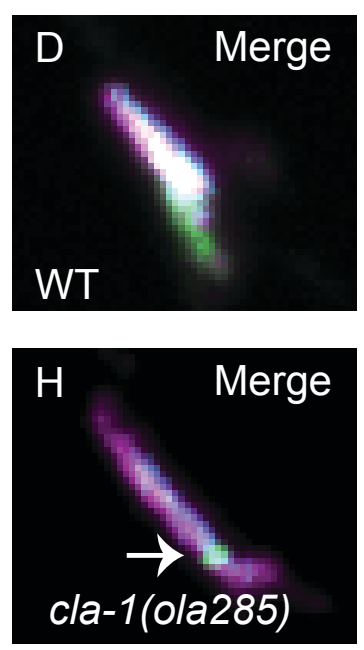
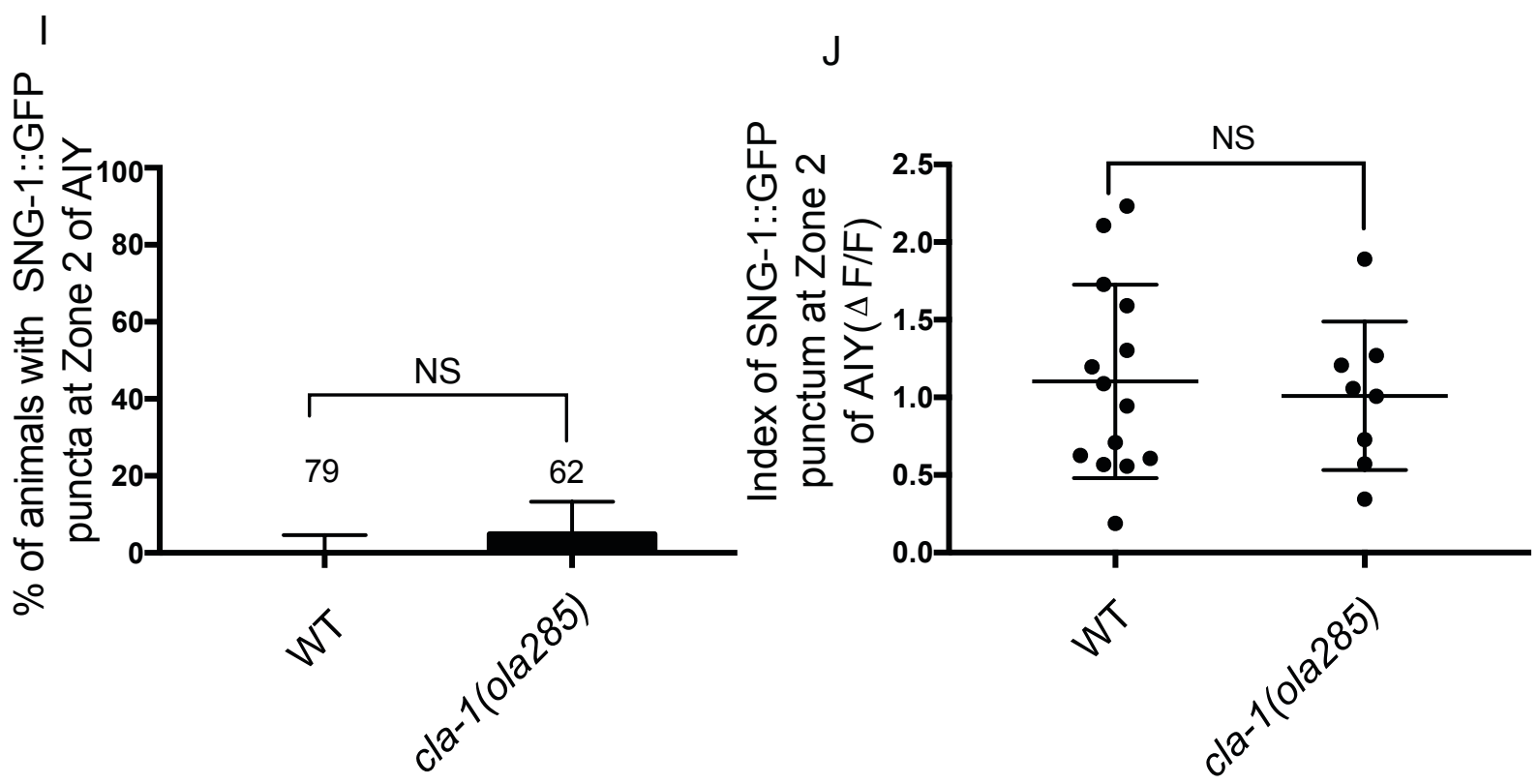

Figure 2 
1098 Figure 2. ATG-9 and synaptic vesicle proteins are differentially regulated by

1099 CLA-1L.

1100 (A-H) Distribution of SNG-1::BFP (pseudo-colored cyan) (A and E), 1101 mCherry::RAB-3 (pseudo-colored magenta) (B and F) and ATG-9::GFP (C and G)

1102 at Zone 2 of AIY (merge in D and H) in wild type (WT) (A-D) and cla-1(ola285)

1103 mutant $(\mathrm{E}-\mathrm{H})$ animals. While we observe a phenotype for abnormal ATG-9

1104 distribution to subsynaptic foci in cla-1(ola285) mutants (indicated by arrows in E-

$1105 \mathrm{H}$ ), we do not see a similar redistribution for synaptic vesicle proteins SNG-1 and

1106 RAB-3.

1107 (I) Quantification of the percentage of animals displaying SNG-1::GFP subsynaptic 1108 foci at AIY Zone 2 in different genotypes. Error bars represent 95\% confidence 1109 interval. "NS" (not significant) by two-tailed Fisher's exact test. The number on the 1110 bars indicates the number of animals scored.

1111 (J) Quantification of the index of SNG-1::GFP punctum $(\Delta F / F)$ at Zone 2 of AIY in 1112 wild type (WT) and cla-1(ola285) mutants. Error bars show standard deviation 1113 (SD). "NS" (not significant) by two-tailed unpaired Student's t-test. Each dot in the 1114 scatter plot represents a single animal.

1115 Scale bar (in A for A-H), $1 \mu \mathrm{m}$. 
1119 Figure 3. ATG-9 accumulates at clathrin-rich foci in cla-1(ola285) mutants

1120 and is suppressed by mutants for synaptic vesicle exocytosis.

1121 (A-H) Distribution of ATG-9::GFP (A and E), BFP::CHC-1 (pseudo-colored cyan)

1122 ( $B$ and $F$ ) and mCherry::RAB-3 (pseudo-colored magenta) ( $C$ and $G$ ) at Zone 2 of

1123 AIY (merge in D and H) in wild type (WT) (A-D) and cla-1(ola285) mutant (E-H)

1124 animals. ATG-9 subsynaptic foci are enriched with CHC-1 in cla-1(ola285) mutants

1125 (indicated by arrows in $\mathrm{E}-\mathrm{H}$ ).

1126 (I) Schematic of the proteins required for the synaptic vesicle cycle and associated

1127 with this study (both the names used for C. elegans and vertebrates are listed).

1128 (J-Q) Distribution of ATG-9::GFP at Zone 2 of AIY in wild type (WT) (J), cla-

1129 1(ola285) (K), unc-13(s69) (L), unc-13(s69);cla-1(ola285) (M), unc-10 (md1117)

1130 (N); unc-10(md1117);cla-1(ola285) (O), unc-18(e81) (P) and unc-18(e81);cla-

1131 1(ola285) (Q) animals. ATG-9 subsynaptic foci are indicated by the arrow (in K).

1132 (R) Quantification of the index of ATG-9 punctum $(\Delta F / F)$ at Zone 2 of AIY in the

1133 indicated genotypes. Error bars show standard deviation (SD). ${ }^{* * * *} p<0.0001$ by

1134 ordinary one-way ANOVA with Tukey's multiple comparisons test. Each dot in the

1135 scatter plot represents a single animal.

1136 Scale bar (in A for A-H and J-Q), $1 \mu \mathrm{m}$. 
C-term labeling of CLA-1
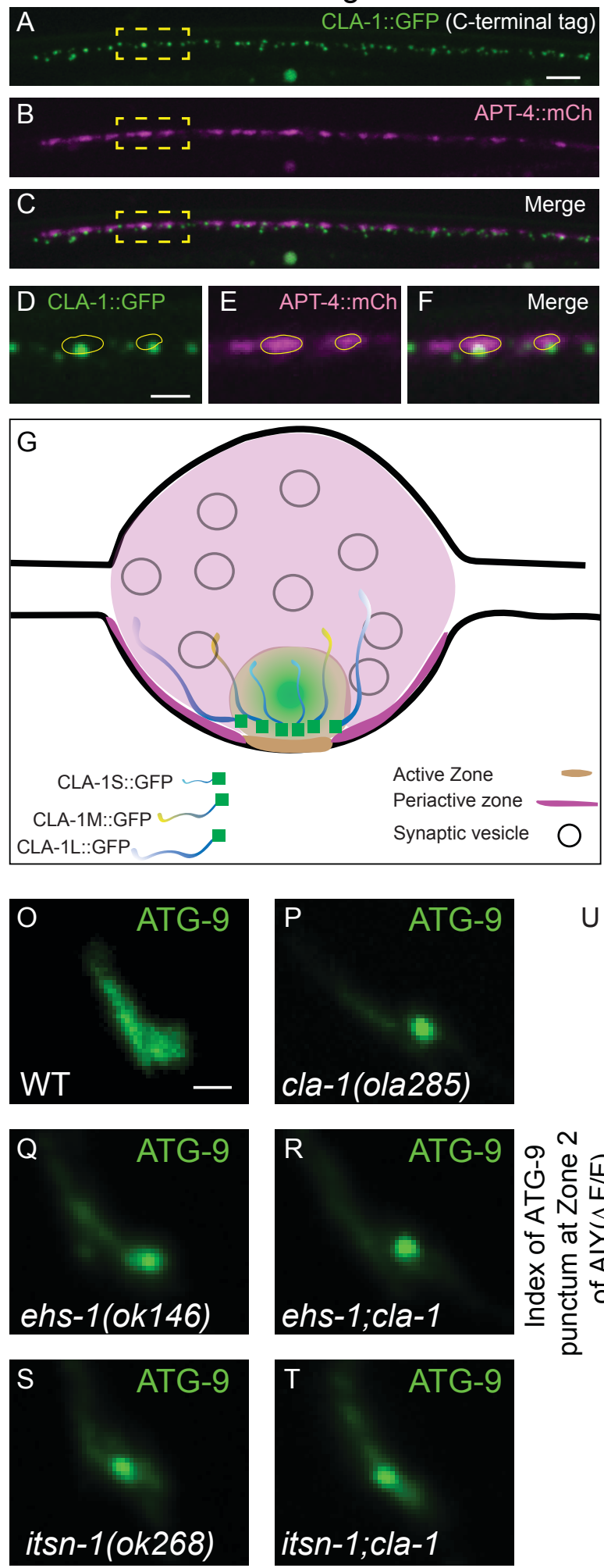

Figure 4
$\mathrm{N}$-term labeling of CLA-1L
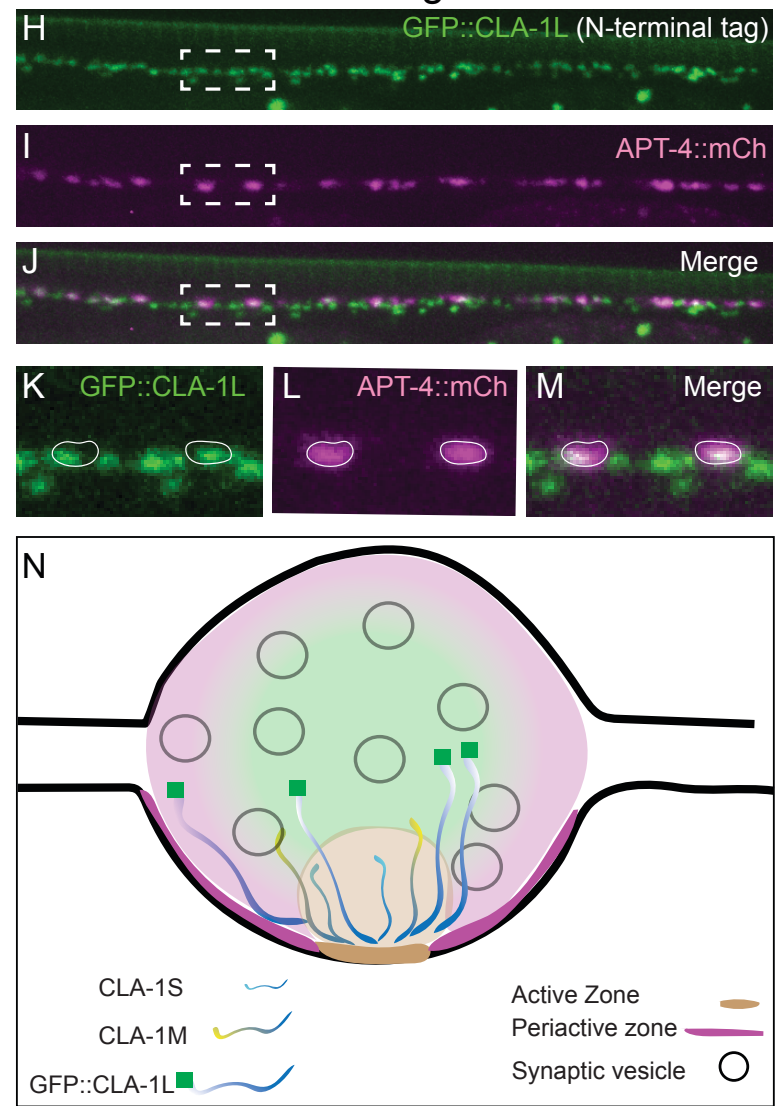

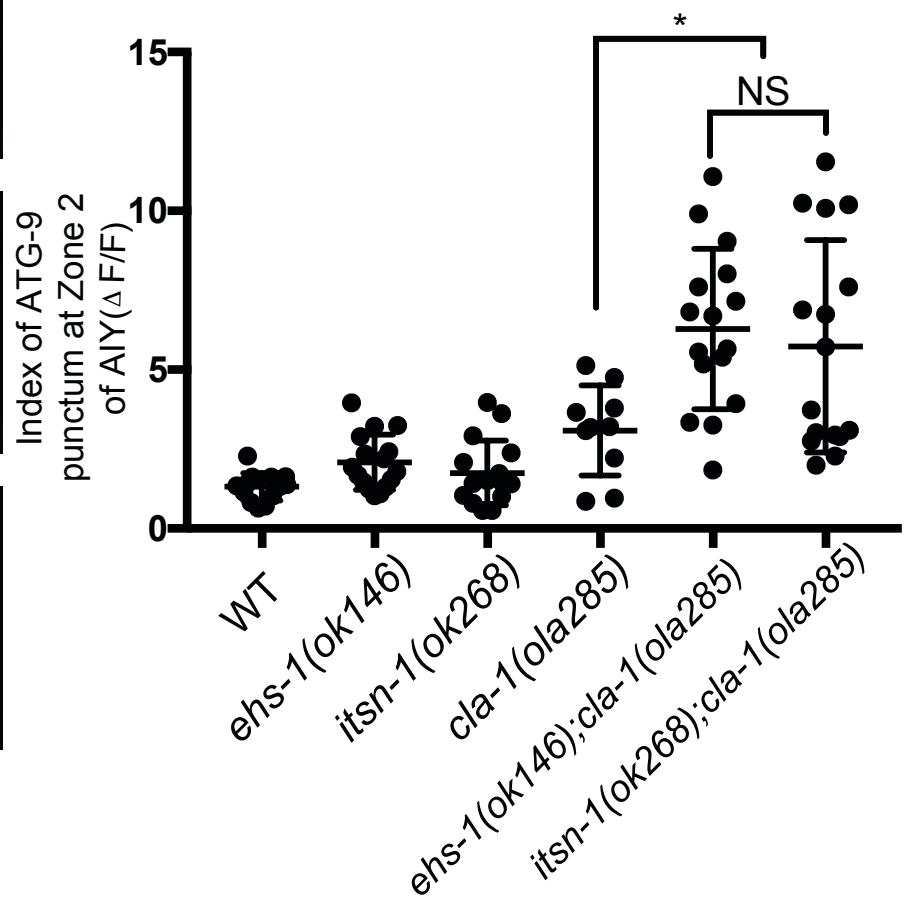


1140 Figure 4. CLA-1L genetically interacts with endocytic proteins at the

1141 periactive zone to regulate ATG-9 trafficking.

1142 (A-C) Distribution of endogenous C-terminally tagged CLA-1::GFP $(A)$ and the

1143 endocytic zone marker APT-4/APA-2/AP-2a::mCherry (APT-4::mCh, pseudo-

1144 colored magenta) (B) in the neurons of the posterior dorsal nerve cord (merge in

$1145 \mathrm{C}$ ) in wild type animals.

1146 (D-F) Enlarged regions enclosed in dashed boxes in A-C. Endogenous C-

1147 terminally tagged CLA-1::GFP (D) localizes to small puncta corresponding to the

1148 active $z o n e^{40}$, and different from the pattern observed for periactive zone protein,

1149 APT-4::mCh (E, merge in F). Yellow circles are drawn based on the outline of APT-

$1150 \quad 4:: m C h$ puncta in $E$, and are located at the same positions in D-F.

1151 (G) Schematic of the localization of the C-terminally tagged CLA-1::GFP, relative

1152 to the subsynaptic active and periactive zones.

$1153(\mathrm{H}-\mathrm{J})$ Distribution of endogenous N-terminally tagged GFP::CLA-1L $(\mathrm{H})$ and the

1154 endocytic zone marker APT-4/APA-2/AP-2a::mCherry (APT-4::mCh, pseudo-

1155 colored magenta) (I) in neurons of the posterior dorsal nerve cord (merge in $\mathrm{J}$ ) in

1156 wild type animals.

1157 (K-M) Enlarged regions enclosed in dashed boxes in $\mathrm{H}-\mathrm{J}$. Endogenous $\mathrm{N}-$ 1158 terminally tagged GFP::CLA-1L (K) displays a more distributed synaptic

1159 distribution as compared to the C-terminally tagged CLA-1:GFP (compare with A,

$1160 \mathrm{D}$ and $\mathrm{F}$, see also ${ }^{40}$ ) and colocalizes with APT-4::mCh (L, merge in $\left.\mathrm{M}\right)$. White

1161 circles are drawn based on the outline of APT-4::mCh puncta in L, and are located

1162 at the same positions in K-M. 
1163 (N) Schematic of the localization of the N-terminally tagged GFP::CLA-1L, relative

1164 to the subsynaptic active and periactive zones.

1165 (O-T) Distribution of ATG-9::GFP at Zone 2 of AlY in wild type (WT) (O), cla-

1166 1(ola285) (P), ehs-1(ok146) (Q), ehs-1(ok146);cla-1(ola285) (R), itsn-1(ok268) (S)

1167 and itsn-1(ok268);cla-1(ola285) (T) mutant animals.

1168 (U) Quantification of the index of ATG-9 punctum $(\Delta \mathrm{F} / \mathrm{F})$ at Zone 2 of AIY in the

1169 indicated genotypes. Error bars show standard deviation (SD). "NS" (not

1170 significant), ${ }^{*} p<0.05$ by ordinary one-way ANOVA with Tukey's multiple

1171 comparisons test. Each dot in the scatter plot represents a single animal.

1172 Scale bar (in A for A-C and H-J), $5 \mu \mathrm{m}$; (in D for D-F and K-M), $2 \mu \mathrm{m}$; (in O for O-

$1173 \mathrm{~T}), 1 \mu \mathrm{m}$.

1174

1175

1176

1177

1178

1179

1180

1181

1182

1183 


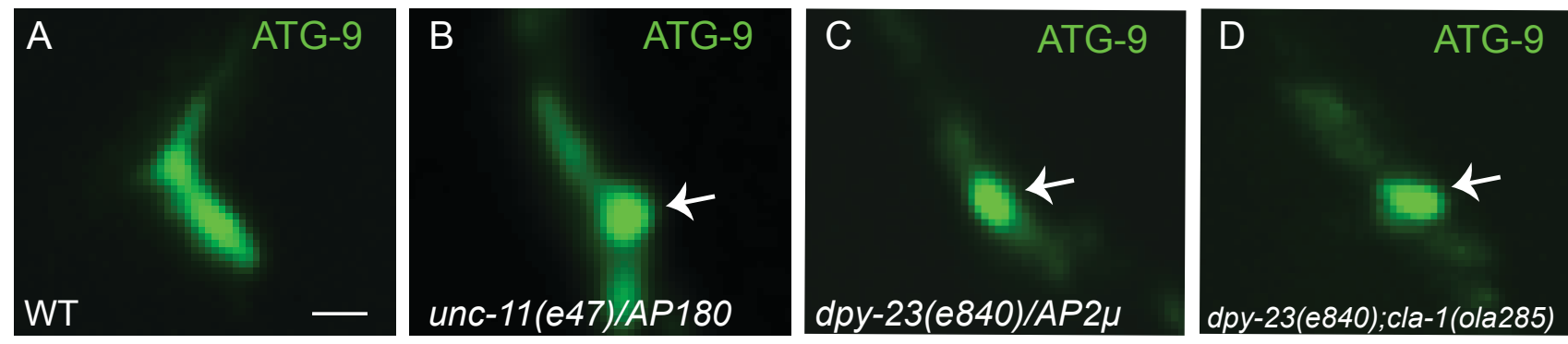

E

$\mathrm{F}$
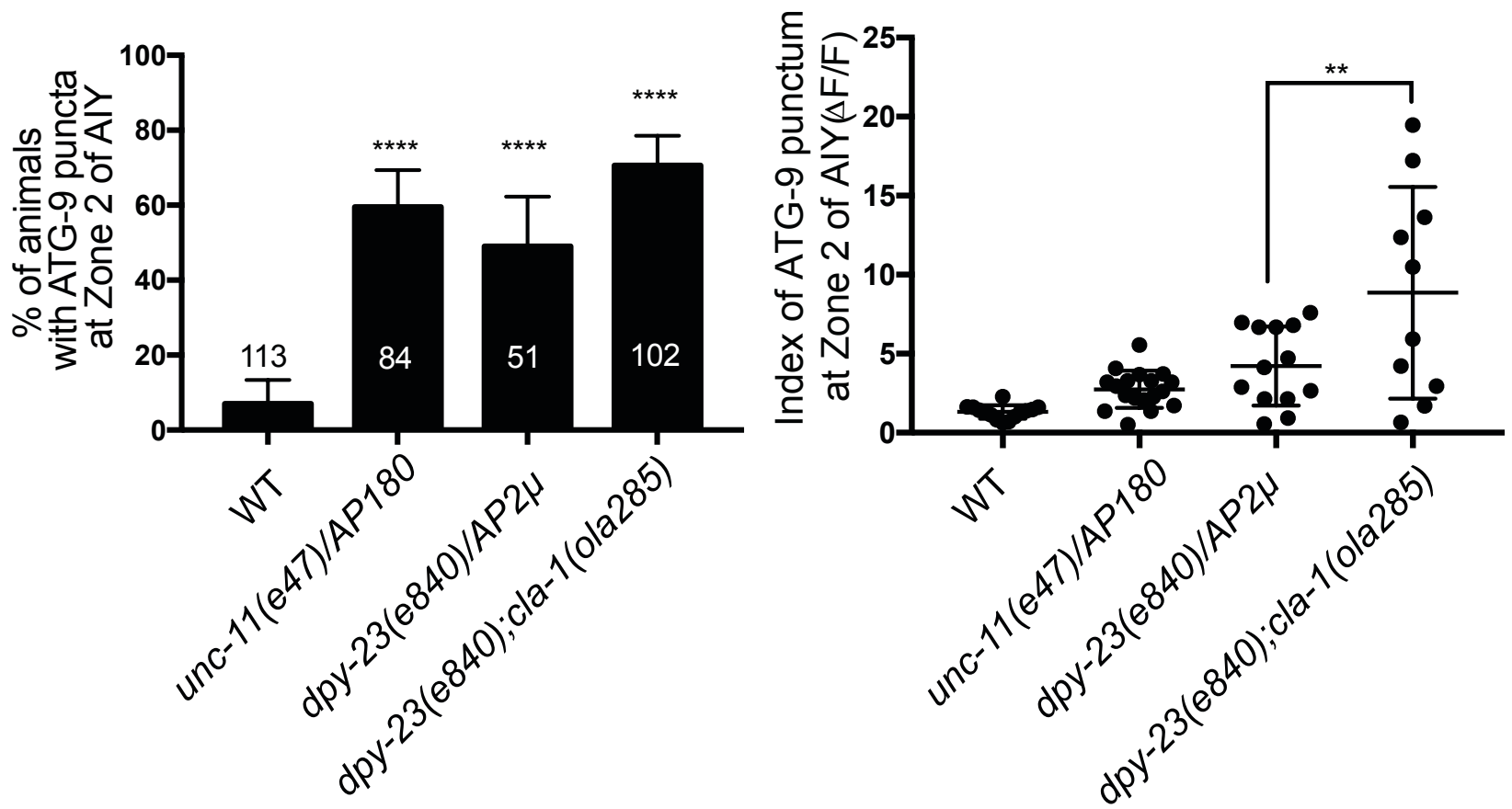

Figure 5 
1184 Figure 5. The clathrin-associated adaptor complexes, AP-2 and AP180,

1185 regulate ATG-9 trafficking at presynaptic sites.

1186 (A-D) Distribution of ATG-9::GFP at Zone 2 of AIY in wild type (WT) (A), unc-

1187 11(e47)/AP180 (B), dpy-23(e840)/AP2 $\mu$ (C) and dpy-23(e840);cla-1(ola285) (D)

1188 mutant animals. Abnormal ATG-9 subsynaptic foci are indicated by arrows in B-D.

1189 (E) Quantification of the percentage of animals displaying ATG-9 subsynaptic foci

1190 at AIY Zone 2 for the indicated genotypes. Error bars represent 95\% confidence

1191 interval. ${ }^{* * *} p<0.0001$ by two-tailed Fisher's exact test. The number on the bars

1192 indicates the number of animals scored.

1193 (F) Quantification of the index of ATG-9 punctum $(\Delta F / F)$ at Zone 2 of AIY for the

1194 indicated genotypes. Error bars show standard deviation (SD). ${ }^{* *} p<0.01$ by

1195 ordinary one-way ANOVA with Tukey's multiple comparisons test. Each dot in the

1196 scatter plot represents a single animal.

1197 Scale bar (in A for A-D), $1 \mu \mathrm{m}$.

1198

1199

1200

1201

1202

1203

1204

1205 


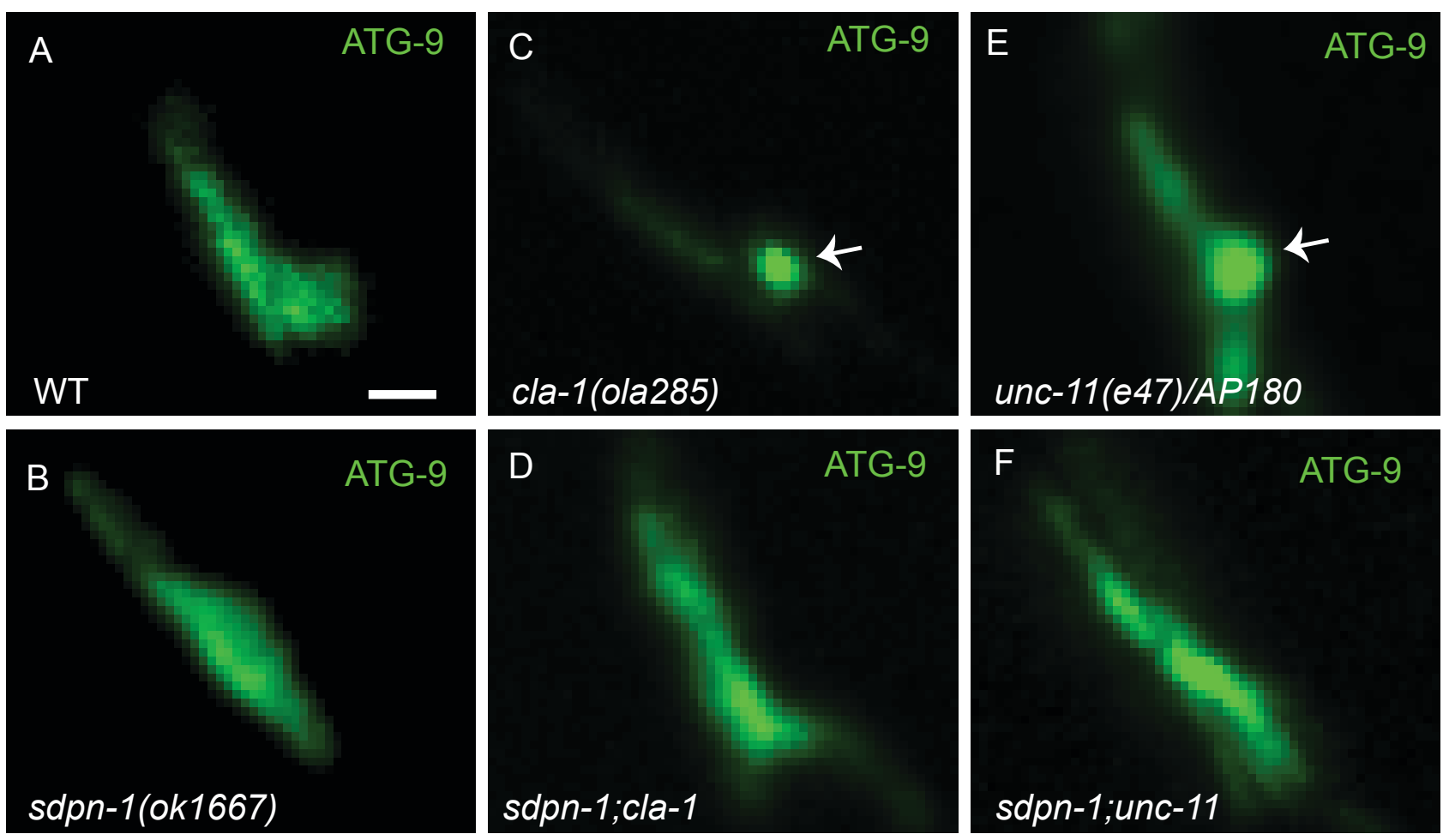

G

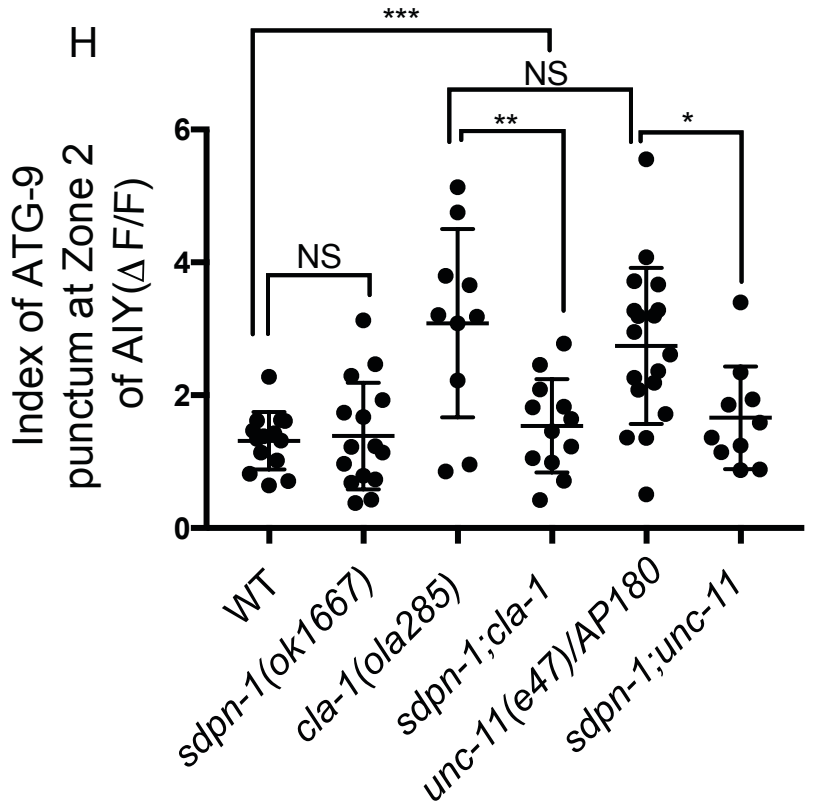

Figure 6 


\section{Figure 6. SDPN-1/syndapin 1 regulates ATG-9 sorting at presynaptic sites}

1207 (A-F) Distribution of ATG-9::GFP at Zone 2 of AIY in wild type (WT) (A), sdpn-

1208 1(ok1667) (B), cla-1(ola285) (C), sdpn-1(ok1667);cla-1(ola285) (D), unc-

1209 11(e47)/AP180 (E) and sdpn-1(ok1667);unc-11(e47) (F) mutant animals.

1210 Abnormal ATG-9 subsynaptic foci are indicated by arrows in C and E. Note that

1211 mutations in SDPN-1/syndapin 1 suppress the abnormal ATG-9 phenotypes in cla-

12121 and unc-11/AP180 mutants.

1213 (G) Quantification of the percentage of animals displaying abnormal ATG-9 1214 subsynaptic foci at AIY Zone 2 for the indicated genotypes. Error bars represent

$121595 \%$ confidence interval. "NS" (not significant), ${ }^{* * *} p<0.0001$ by two-tailed Fisher's

1216 exact test. The number on the bars indicates the number of animals scored.

$1217(\mathrm{H})$ Quantification of the index of ATG-9 punctum $(\Delta \mathrm{F} / \mathrm{F})$ at Zone 2 of AIY for the

1218 indicated genotypes. Error bars show standard deviation (SD). "NS" (not

1219 significant), ${ }^{*} p<0.05,{ }^{* *} p<0.01,{ }^{* * *} p<0.001$ by ordinary one-way ANOVA with

1220 Tukey's multiple comparisons test. Each dot in the scatter plot represents a single

1221 animal.

1222 Scale bar (in A for A-F), $1 \mu \mathrm{m}$. 


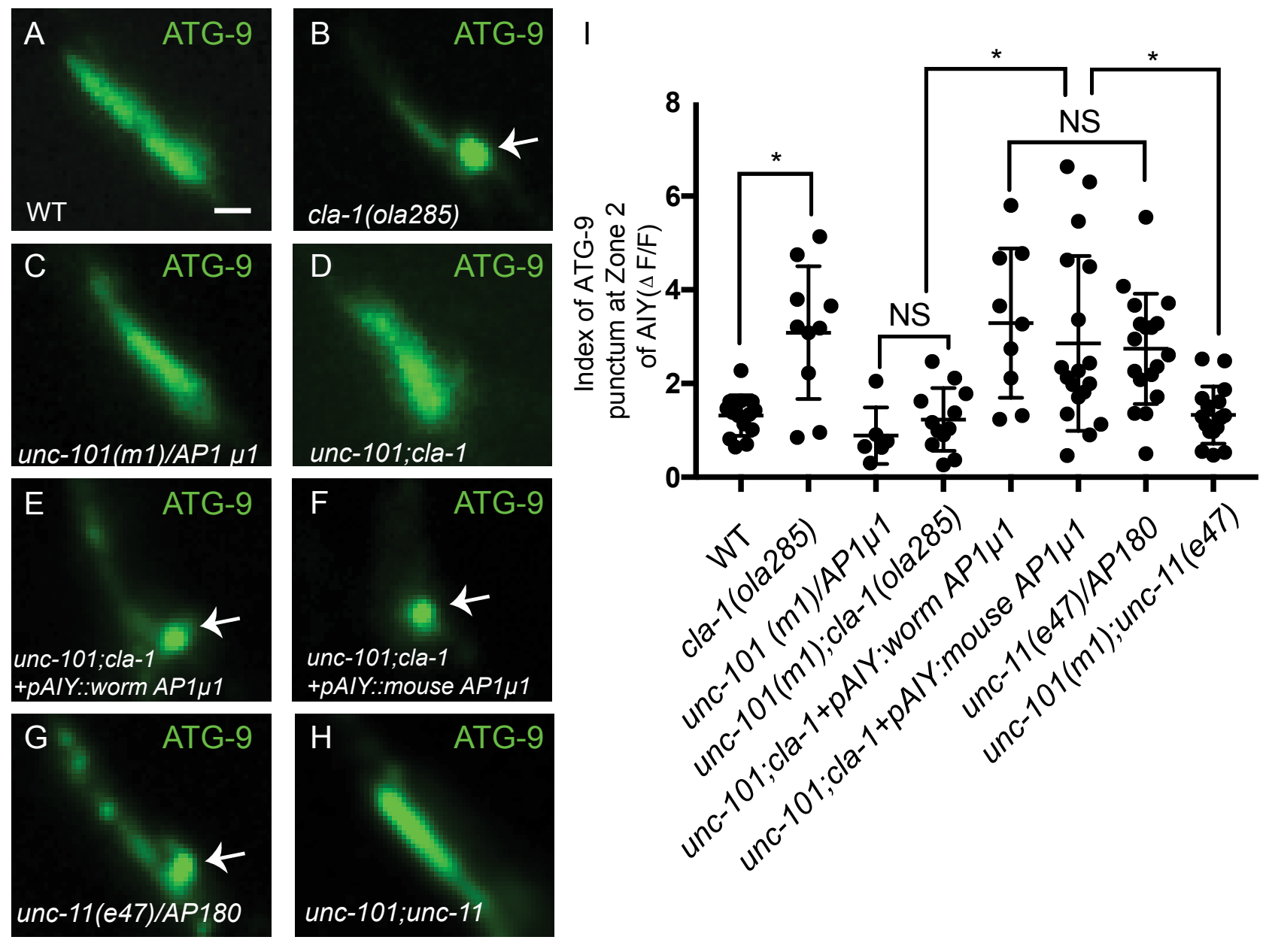

$J$

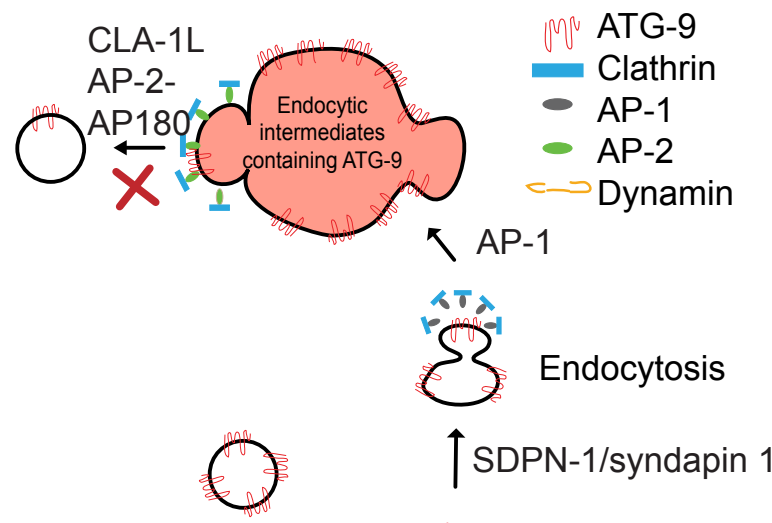

Exocytosis $\downarrow$

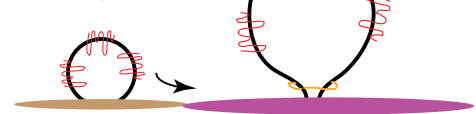

Active Zone Periactive zone

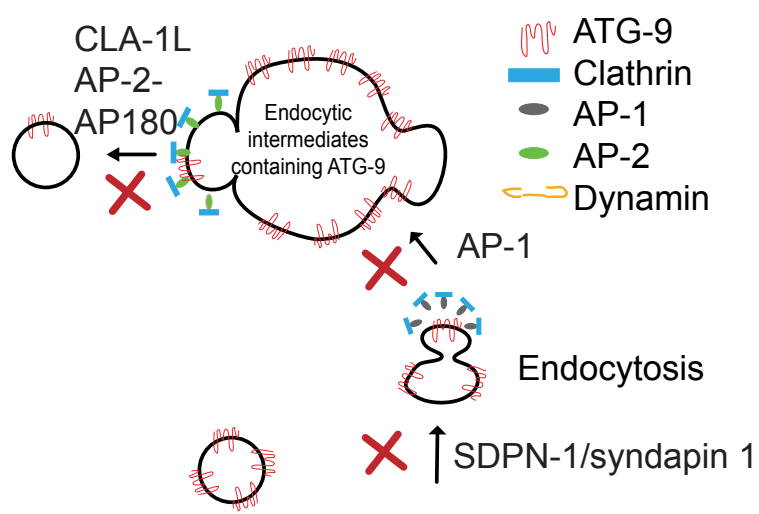

Exocytosis $\downarrow$

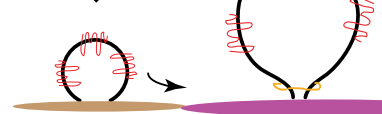

Active Zone Periactive zone

Figure 7 
1228 Figure 7. ATG-9 is sorted to the endocytic intermediates via the AP-1 adaptor

1229 complex.

1230 (A-H) Distribution of ATG-9::GFP at Zone 2 of AIY in wild type (WT) (A), cla-

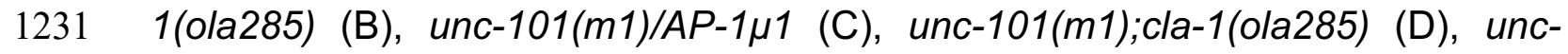

1232 101;cla-1 mutants with C. elegans UNC-101/AP-1 $\mu 1$ cDNA cell-specifically 1233 expressed in AIY (E), unc-101;cla-1 mutants with mouse AP1 1 cDNA cell1234 specifically expressed in AIY (F), unc-11(e47)/AP180 (G) and unc-101(m1);unc1235 11(e47) (H). Abnormal ATG-9 subsynaptic foci are indicated by arrows in B and E1236 G.

1237 (I) Quantification of the index of ATG-9 punctum $(\Delta \mathrm{F} / \mathrm{F})$ at Zone 2 of AIY for 1238 indicated genotypes. Error bars show standard deviation (SD). "NS" (not 1239 significant), ${ }^{*} p<0.05$ by ordinary one-way ANOVA with Tukey's multiple 1240 comparisons test. Each dot in the scatter plot represents a single animal.

1241 (J) Cartoon diagram of the genetic interactions, and model, in this study. Mutants 1242 for CLA-1L, AP-2 and AP180 adaptor complexes display similar ATG-9 1243 phenotypes at synapses, and are necessary for resolving ATG-9-containing foci 1244 (clathrin rich endocytic intermediates).

1245 (K) Cartoon diagram of the genetic interactions, and model displaying the genetic 1246 interactions of CLA-1L with SDPN-1/syndapin 1 and the AP-1 adaptor complex, 1247 which suppress the ATG-9 phenotypes observed for cla-1(L), AP-2 or AP180 1248 mutants, consistent with roles for SDPN-1/syndapin 1 and the AP-1 upstream of 1249 the formation of the abnormal ATG-9 foci (similar to what was observed for 
bioRxiv preprint doi: https://doi.org/10.1101/2021.08.19.457026; this version posted August 19, 2021. The copyright holder for this preprint

(which was not certified by peer review) is the author/funder, who has granted bioRxiv a license to display the preprint in perpetuity. It is made available under aCC-BY-NC-ND 4.0 International license.

1250 exocytosis mutants in Figure 3, which also suppressed ATG-9 phenotypes in cla-

$12511(L)$ mutants).

1252 Scale bar (in A for A-H), $1 \mu \mathrm{m}$.

1253

1254

1255

1256

1257

1258

1259

1260

1261

1262

1263

1264

1265

1266

1267

1268

1269 

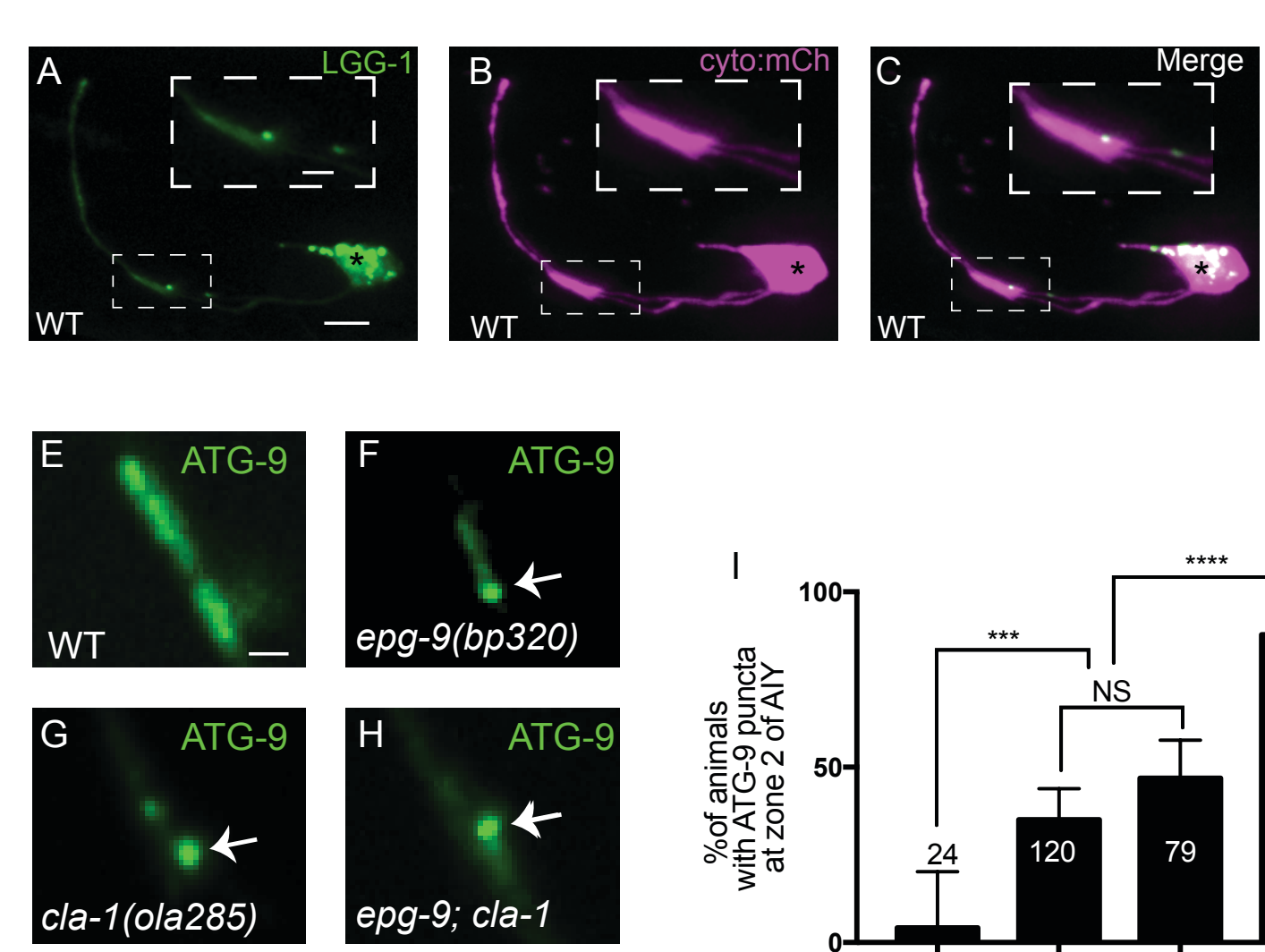
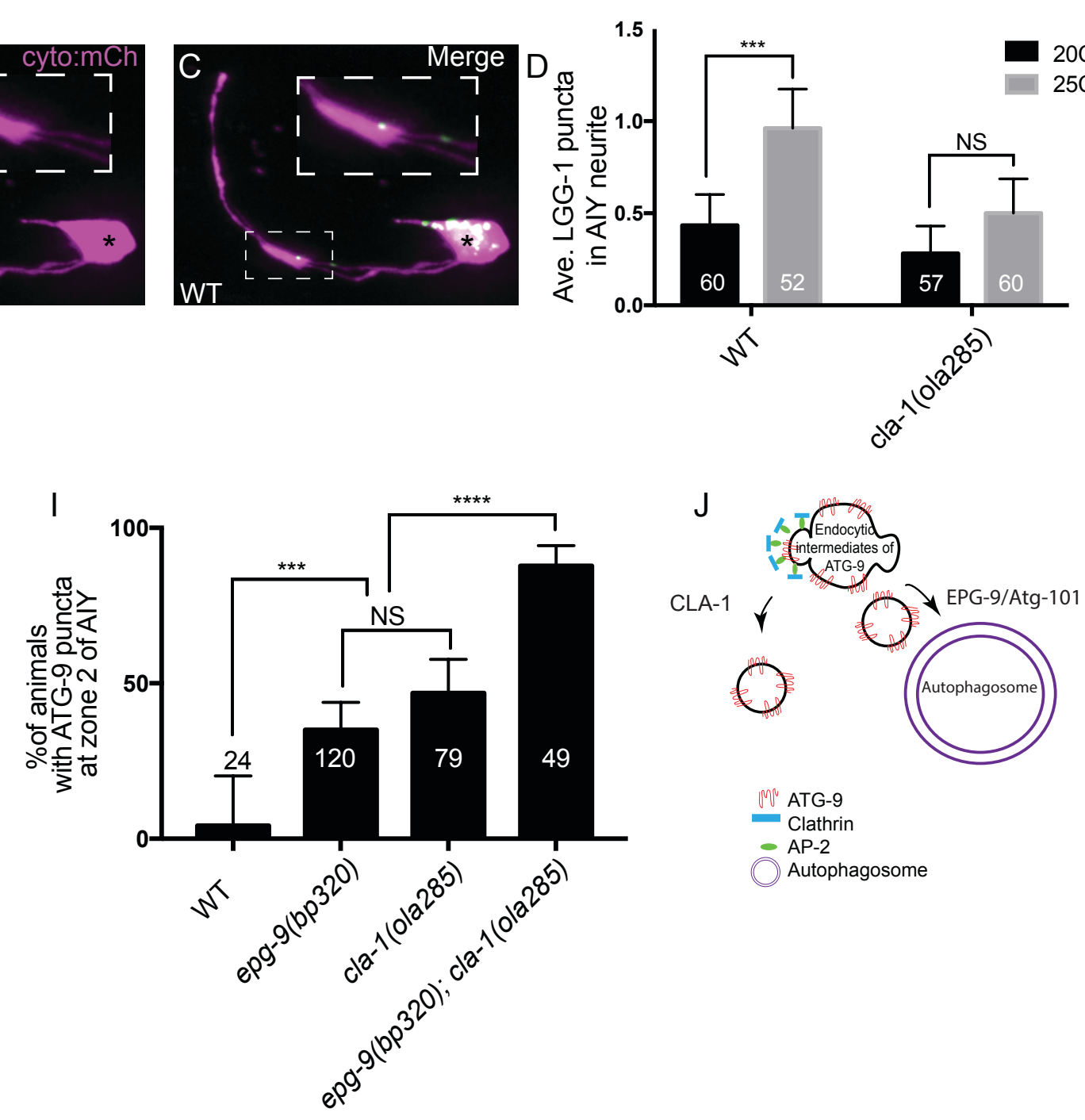

- AP-2

Autophagosome

Figure 8 
1270 Figure 8. Disrupted ATG-9 trafficking in cla-1(L) mutants is associated with

1271 a deficit in activity-induced autophagosome formation.

1272 (A-C) Confocal micrographs of GFP::LGG-1 (A) and cytoplasmic mCherry

1273 (cyto::mCh) (pseudo-colored magenta, B) in AIY (merge in C). Inset is the enlarged

1274 region enclosed in dashed box to show one LGG-1 punctum in AIY synaptic Zone

12752.

1276 (D) Quantification of the average number of LGG-1 puncta in the AIY neurites at

$127720^{\circ} \mathrm{C}$ and at $25^{\circ} \mathrm{C}$ for 4 hours in wild type (WT) and cla-1(ola285) mutants. (Note:

1278 The activity state of the thermotaxis interneurons AIY is reported to increase when

1279 animals are cultivated at $25^{\circ} \mathrm{C}$ for 4 hours, compared to animals at $20^{\circ} \mathrm{C}^{95,139,140 .}$

1280 Error bars represent 95\% confidence interval. "NS" (not significant), ${ }^{* * *} p<0.001$ by

1281 Kruskal-Wallis test with Dunn's multiple comparisons test. The number on the bars

1282 indicates the number of animals scored.

$1283(\mathrm{E}-\mathrm{H})$ Distribution of ATG-9::GFP at Zone 2 of AIY in wild type (E), epg-9(bp320)

1284 (F), cla-1(ola285) (G) and epg-9(bp320); cla-1(ola285) (H) mutant animals. Arrows

1285 (in F-H) indicate abnormal ATG-9 foci.

1286 (I) Quantification of the percentage of animals displaying ATG-9 subsynaptic foci

1287 at AIY Zone 2 in the indicated genotypes. Error bars represent $95 \%$ confidence

1288 interval. "NS" (not significant), ${ }^{* *} p<0.001,{ }^{* * *} p<0.0001$ by two-tailed Fisher's

1289 exact test. The number on the bar indicates the number of animals scored.

1290 (J) Cartoon diagram representing autophagosome biogenesis at the synapse, and

1291 our model on its relationship with ATG-9 trafficking. The ATG-9 phenotype is

1292 enhanced in epg-9(bp320); cla-1(ola285) double mutants. 
bioRxiv preprint doi: https://doi.org/10.1101/2021.08.19.457026; this version posted August 19, 2021. The copyright holder for this preprint (which was not certified by peer review) is the author/funder, who has granted bioRxiv a license to display the preprint in perpetuity. It is made available under aCC-BY-NC-ND 4.0 International license.

1293 Scale bar (in A for A-C), $5 \mu \mathrm{m}$; (in inset of $A$ for inset of $A-C$ ), $2 \mu m$; (in E for $E-H$ ),

$12941 \mu \mathrm{m}$.

1295

1296

1297

1298

1299

1300

1301

1302

1303

1304

1305

1306

1307

1308

1309

1310

1311

1312 


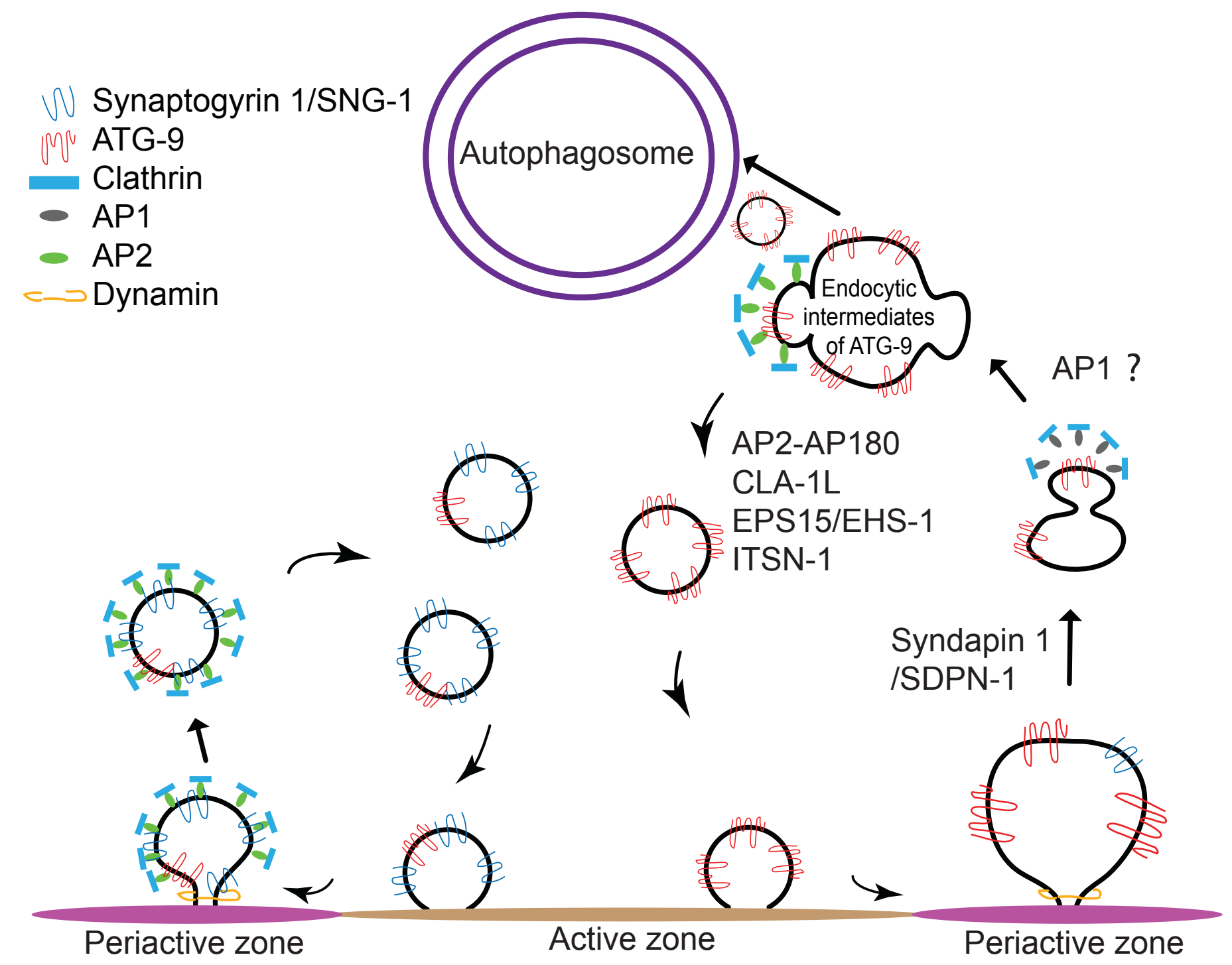

Figure 9 
1313 Figure 9. Cartoon diagram representing the genetic relationships between

1314 ATG-9 trafficking, the synaptic vesicle cycle and synaptic autophagy.

1315 At Zone 2 of AIY, both synaptic vesicles and ATG-9 vesicles undergo exo-

1316 endocytosis ${ }^{21}$. While most synaptic vesicles undergo clathrin-mediated

1317 endocytosis, our data are consistent with ATG-9 undergoing endosome-mediated

1318 endocytosis and displaying distinct phenotypes than those seen for synaptic

1319 vesicle proteins. We propose a model whereby ATG-9 is sorted by the adaptor

1320 complex AP1 to the endosome-like structures. CLA-1L, together with presynaptic

1321 endocytic proteins that reside in the periactive zone, such as EHS-1 and ITSN-1,

1322 as well as the adaptor complexes such as AP-2 and AP180, are necessary for

1323 sorting of ATG-9 from endocytic intermediates. Mutations in the active zone gene

1324 cla-1L result in abnormal accumulation of ATG-9 into endocytic intermediates, and

1325 defects in activity-dependent autophagosome formation. 

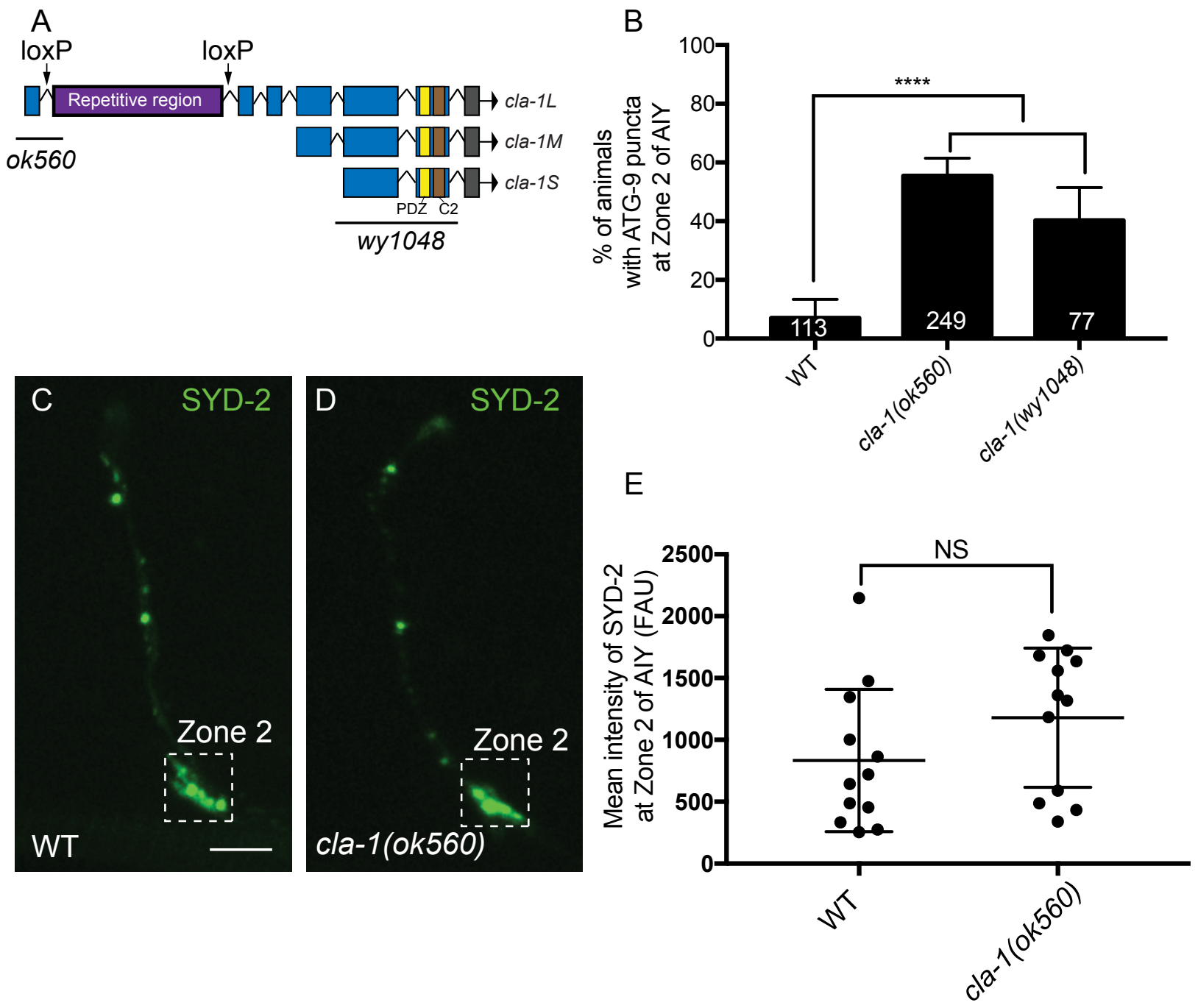

Supplemental figure 1 
1335 Supplemental figure 1. Examination of the active zone protein SYD-2 in cla-

1336 1(L) mutants, and of ATG-9 distribution in cla-1(wy1048) null allele.

1337 (A) Schematic of cla-1 gene, with different protein isoforms. The ok560 allele 1338 specifically affects the long protein isoform, while wy1048 allele affects all CLA-1

1339 protein isoforms.

1340 (B) Quantification of the percentage of animals displaying ATG-9 subsynaptic foci

1341 at AIY Zone 2 in the indicated genotypes. Error bars represent 95\% confidence

1342 interval. ${ }^{* * *} p<0.0001$ by two-tailed Fisher's exact test. The number on the bars

1343 indicates the number of animals scored.

1344 (C-D) Distribution of SYD-2::GFP at the synaptic Zone 2 and Zone 3 regions of

1345 AIY in wild type (C) and cla-1(ok560) (D) animals. The dashed boxes highlight the 1346 presynaptic Zone 2 of AIY examined in this study.

1347 (E) Mean intensity of SYD-2 at AIY Zone 2 in wild type (WT) and cla-1(ok560) 1348 mutants. Error bars show standard deviation (SD). "NS" (not significant) by two1349 tailed unpaired Student's t-test. Each dot in the scatter plot represents a single 1350 animal.

1351 Scale bar (in C for C-D), $5 \mu \mathrm{m}$. 

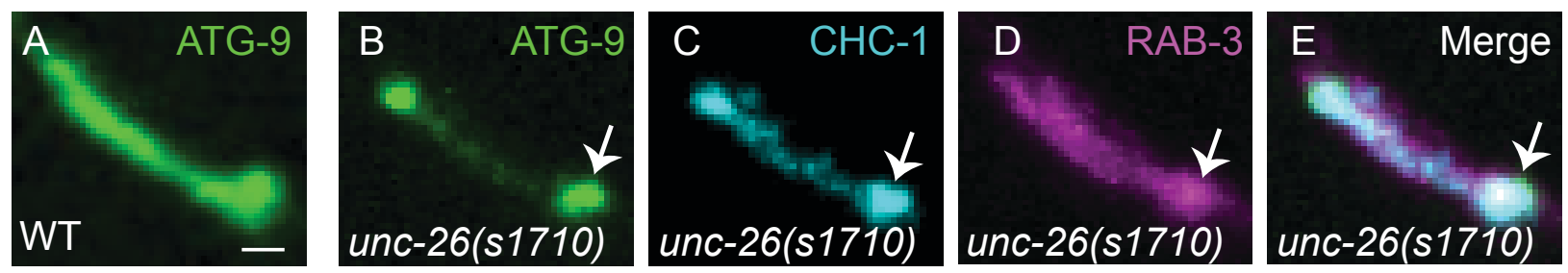

$\mathrm{F}$

竞

ช $\sim$ है

은 은 $\frac{E}{2} 0.6-$

N N

ป స

웅

రํำ $\frac{1}{\square} \frac{1}{0} 0.2-$

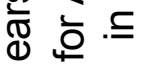

口
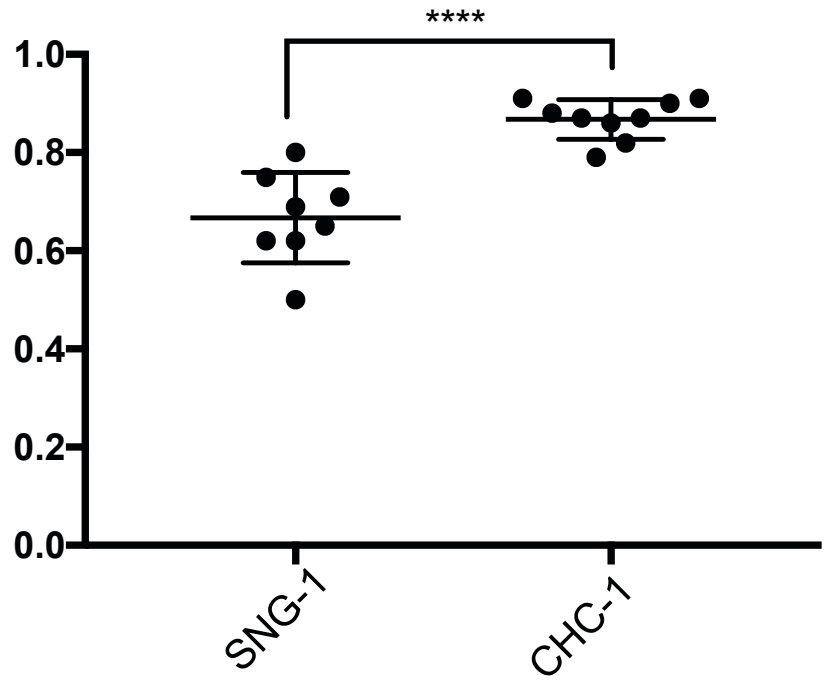

G
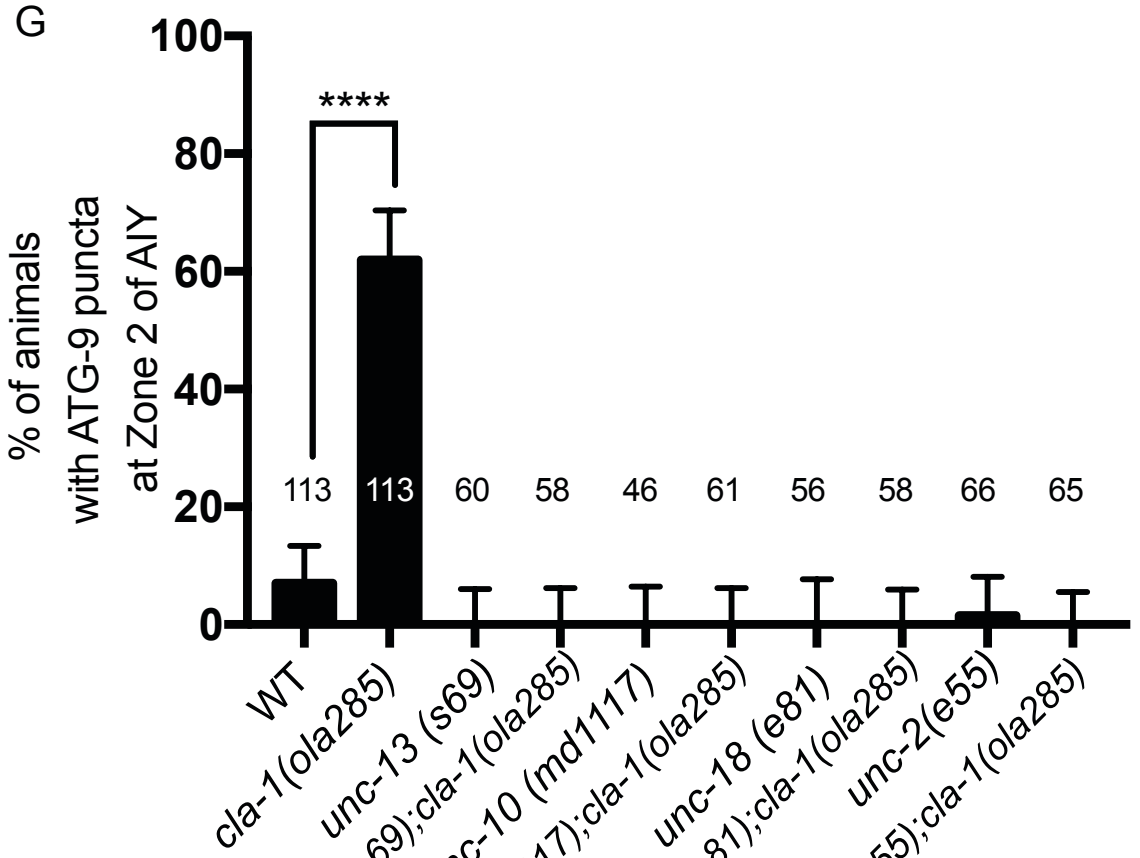

Supplemental figure 2

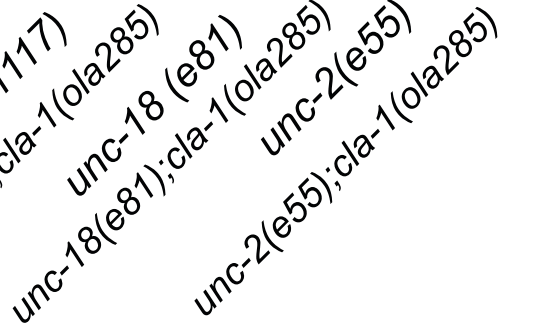


1356 Supplemental figure 2. ATG-9 accumulates at clathrin-rich foci in cla-

1357 1(ola285) mutants, which is suppressed by mutants for synaptic vesicle 1358 exocytosis.

1359 (A) Distribution of ATG-9::GFP at Zone 2 of AIY in wildtype (WT) animals (A).

1360 (B-E) Distribution of ATG-9::GFP (B), BFP::CHC-1 (pseudo-colored cyan) (C) and

1361 mCherry::RAB-3 (pseudo-colored magenta) (D) at Zone 2 of AIY (merge in E) in

1362 26(s1710)/synaptojanin 1 mutant animals (see also ${ }^{21}$ ). ATG-9 subsynaptic foci

1363 (indicated by arrows in B-E) are enriched with $\mathrm{CHC}-1$ in unc1364 26(s1710)/synaptojanin 1 mutants.

1365 (F) Pearson correlation coefficient for colocalization between ATG-9::GFP and

1366 SNG-1::BFP ,or between ATG-9::GFP and BFP::CHC-1, both in cla-1(ola285)

1367 mutants. Error bars show standard deviation (SD). ${ }^{* * *} p<0.0001$ by two-tailed

1368 unpaired Student's t-test. Each dot in the scatter plot represents a single animal.

1369 (G) Quantification of the percentage of animals displaying ATG-9 subsynaptic foci

1370 at AIY Zone 2 in the indicated genotypes. Error bars represent $95 \%$ confidence

1371 interval. ${ }^{* * *} p<0.0001$ by two-tailed Fisher's exact test. The number on the bars

1372 indicates the number of animals scored.

1373 Scale bar (in A for A-E), $1 \mu \mathrm{m}$. 
A

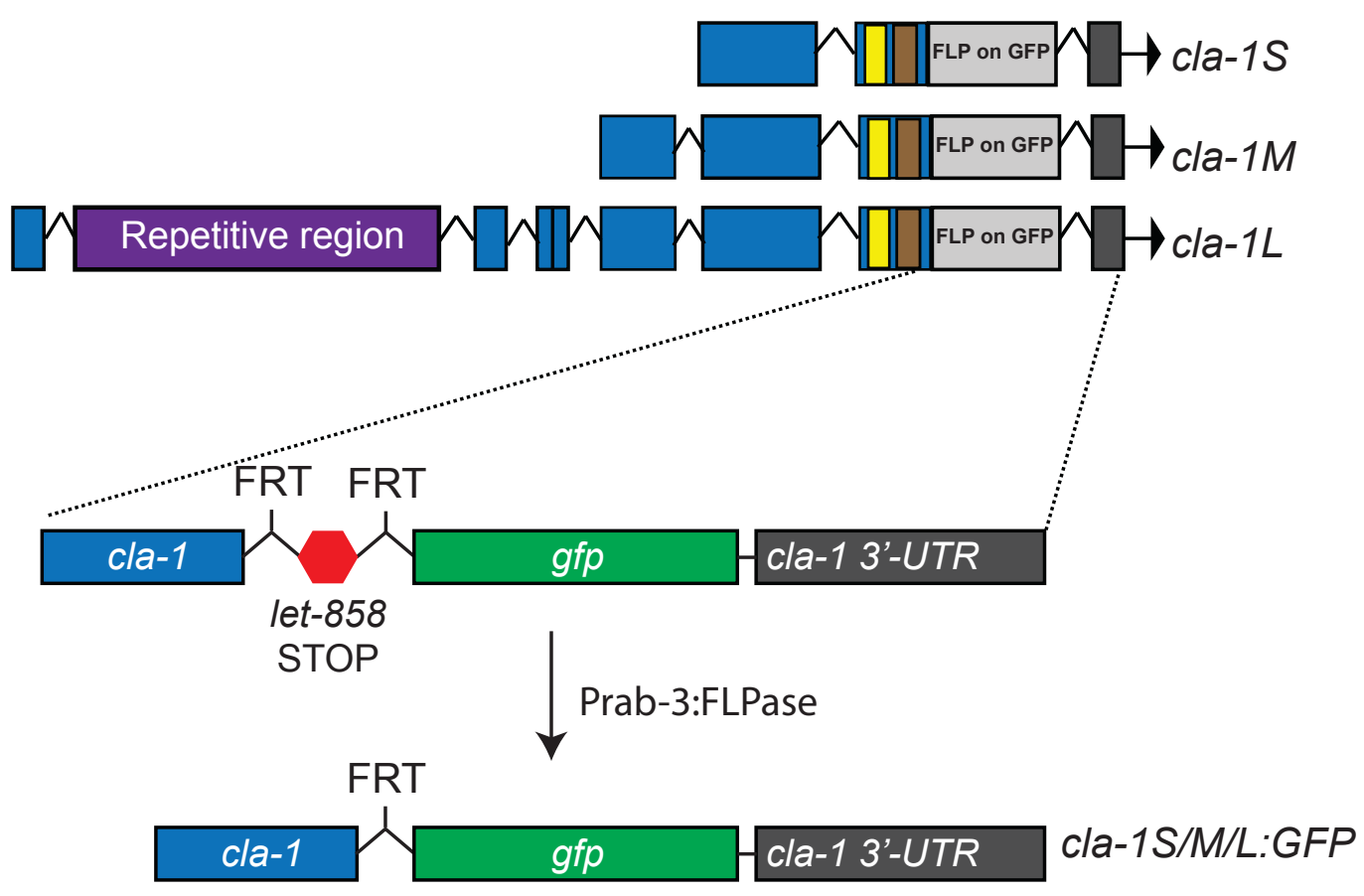

B

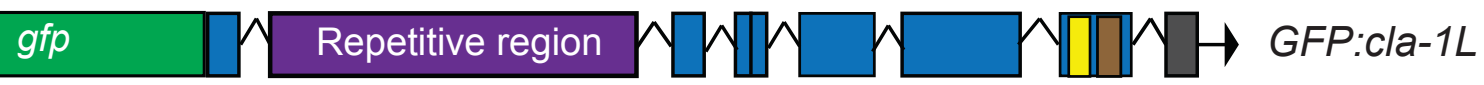

C

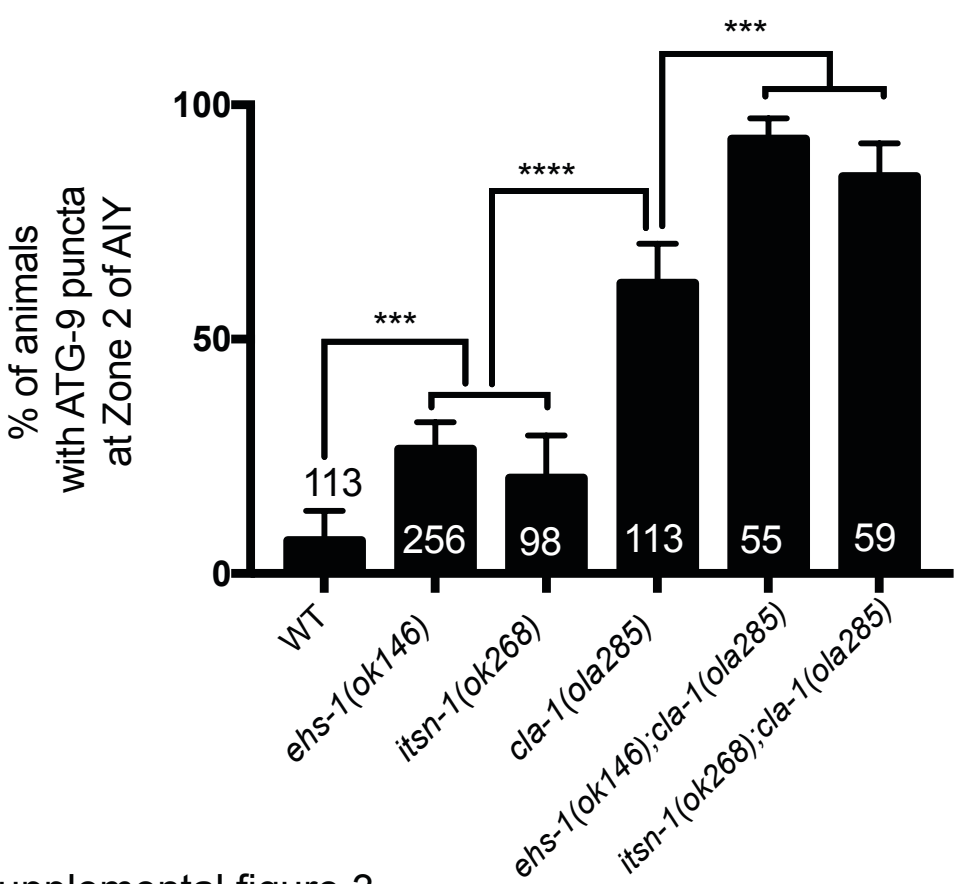

Supplemental figure 3 
1377 Supplemental figure 3. CLA-1L genetically interacts with the endocytic

1378 proteins at the periactive zone to regulate ATG-9 trafficking.

1379 (A) Schematics of the strategy for endogenously tagging CLA-1 at C-terminus via

1380 CRISPR. FLP-on-GFP (let-858 3'UTR flanked by FRT sites followed by GFP) was

1381 inserted at the common C-terminus of CLA-1 (shared by CLA-1L, CLA-1M and

1382 CLA-1S protein isoforms, see ${ }^{40}$ ). FLPase driven by the Prab-3 promoter is 1383 expressed pan-neuronally to induce expression of CLA-1::GFP in an endogenous 1384 manner (see Figure 4A).

1385 (B) Schematics of endogenously tagging CLA-1L at N-terminus via CRISPR ${ }^{40 .}$ 1386 GFP was inserted at the unique N-terminus of CLA-1L (see Figure $4 \mathrm{H}$ ).

1387 (C) Quantification of the percentage of animals displaying ATG-9 subsynaptic foci 1388 at AIY Zone 2 in the indicated genotypes. Error bars represent $95 \%$ confidence 1389 interval. ${ }^{* *} p<0.001,{ }^{* * * *} p<0.0001$ by two-tailed Fisher's exact test. The number on 1390 the bars indicates the number of animals scored. 


\section{A}

worm AP1 1 1 MATSAMF I LDLKGKT I I SRNYRGDI DMTA I DKFI HLLMEKEEEGSAAPVLTYQDTNFVF IKHTNIYLVSACRSNVNVTM I 80

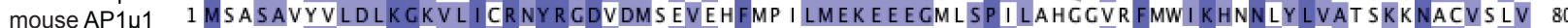
mouse AP2 1 MI - GGLF IYNHKGEVLI ISRVYRDDI GRNAVDAERVNVI HARQ-QVR SPVTNIARTSEFHVKR SN I WLAAVTKQNVNAAMV 78

81 LSF LYKCVEVFSEYFKDVEE E SVR DNFVVIYELLDEMMDFGFPQTTESR I LQEY I TQEGQKLIS-APR- - PPMAVTNAVS 157 81 F SF LYKVVQVFSEY FKELEEESIRDNFV I IYELLDE LMDFGYPQTTDSKI LQEY I TQEGHKLETGAPR - - PPATVTNAVS 158

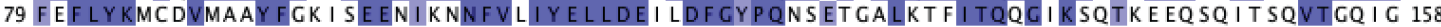

Worm AP1 1158 WR SEG I KYRKNEVF LDV I ESVNMLASANGTVLQSE I VG SVKMRVYLTGMPELR LGLNDKVLFEGSGRGKS- - - - - - KS 229

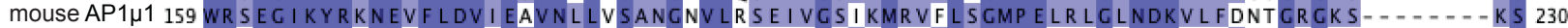
mouse AP2 159 WRRECI KYRRNELF LDVLESVNL LMSPQGQVLSAHV SCRVVMK SYLSCMP ECK FCMNDK IV I EKQCKGTADETSKSGKQS 238

230 VE LEDVKFHQCVR LSR FDTDRT I S F I P P DGAFELMSYR LTTVVKP L I W I ETSI ER HSHSRVSF I IKAKSQFKRRSTANNV 309 231 VELEDVK FHQCVR LSR FENDRT I S F I P P DGEFELMSYR LNTHVKP L I W I ESVI EKHSHSR I EYMVKAKSQFKRRSTANNV 310 239 I A I DDCTFHQCVR LSKFDSERSI SF I PPDGEFELMRYRTTKDI I LPFRV I L LVREVGRTKLEVKVVIKSNFKPSLLAQK I 318

Worm AP1 1310 EI I I PVPSDADSP K FKTS I GSVKYTP EQSAFVWT I KNFP GCKEYLLTAHLSLP SVMSEESEGRPP I KVKFE IPYFTTSG I 389 mouse AP1 1311 E I H I PVPNDADSPK FKTTVG SVKWVPENSE I VWSVKSFPCCKEYLMRAHFGLP SVEAEDKEGKPP I SVKFE IPYFTTSC I 390 mouse AP2 319 EVR I P TP LNT SGVQV I CMKGKAKYKASENA I VWK I KRMAGMKESQ I SAE I ELLP TNDKKKWARPP I SMNFEVP-FAP S GL 397

390 QVRY LK I I EK- - - - SCYQALPWVRY I TQNCEYEMRMK

391 QVRYLK I I EK- - - SCYQALPWVRY I TQNCDYQLRTQ

398 KVRY LKVFEPKLNY SDHDV I KWVRY I GR SC I YETRC-
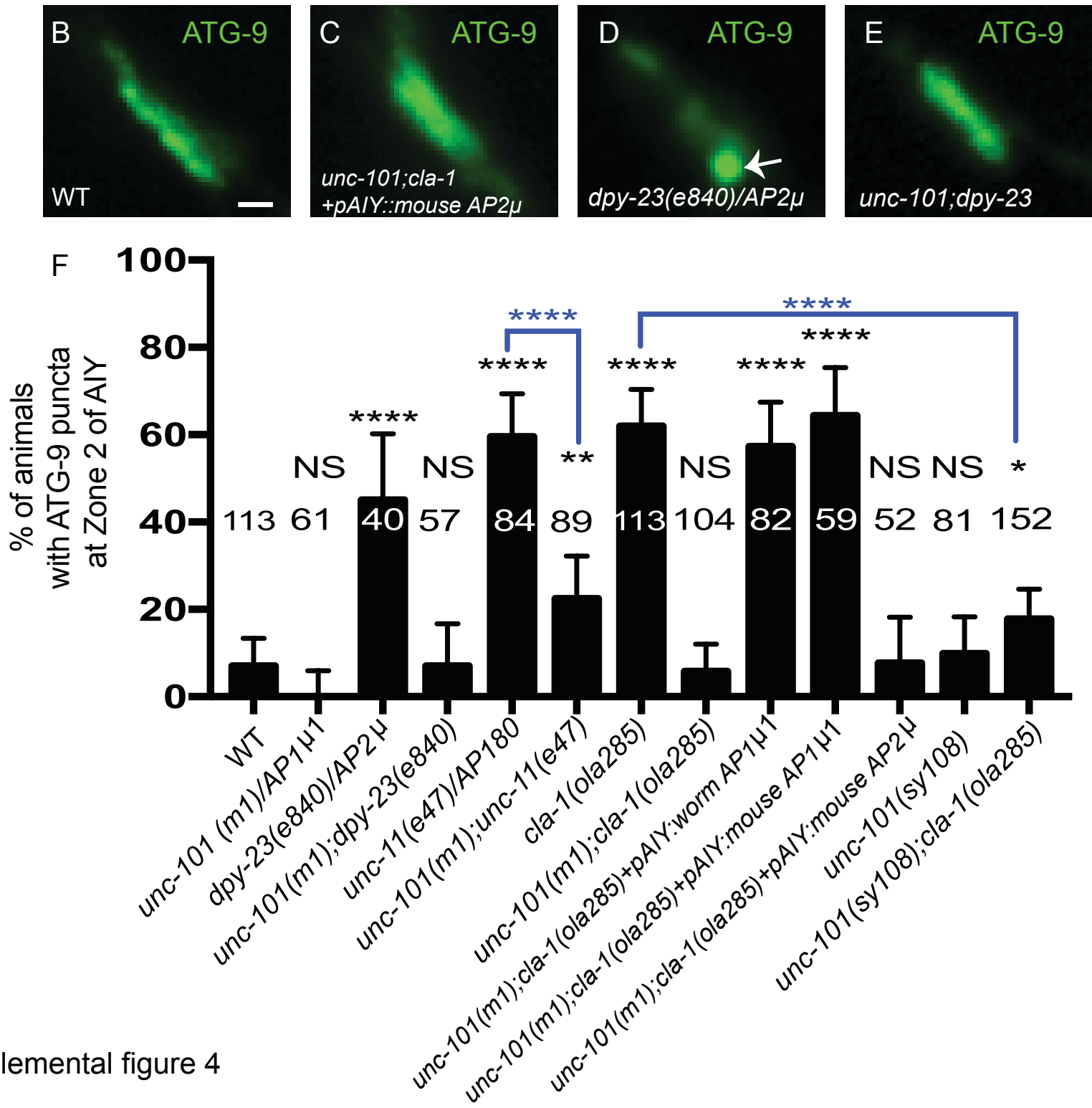
1397 Supplemental figure 4. The AP-1 and AP-2 adaptor complexes mediate

1398 presynaptic trafficking of ATG-9.

1399 (A) Sequence alignment of C. elegans UNC-101/AP1 1 , mouse AP1 1 1 and 1400 mouse AP2 $\mu$, generated using Tcoffee in Jalview ${ }^{137}$. Although both AP-1 $\mu 1$ and 1401 AP- $2 \mu$ share similarity, $C$. elegans UNC-101/AP1 $\mu 1$ is more similar to the mouse 1402 AP1 $\mu 1$ (Query Cover: 100\%; Percentage Identity: 74\%) than the mouse AP2 $\mu$ 1403 (Query Cover: 98\%; Percentage Identity: 41\%).

1404 (B-E) Distribution of ATG-9::GFP at Zone 2 of AIY in wild type (WT) (B), unc1405 101(m1);cla-1(ola285) mutants with mouse AP2 $\mu$ cDNA cell-specifically expressed 1406 in AIY (C), dpy-23(e840)/AP2 $\mu$ (D) and unc-101(m1);dpy-23(e840) (E) mutant 1407 animals. ATG-9 subsynaptic foci are indicated by the arrow (in D).

1409 (F) Quantification of the percentage of animals displaying ATG-9 subsynaptic foci 1410 at AIY Zone 2 in the indicated genotypes. Error bars represent 95\% confidence 1411 interval. "NS" (not significant), ${ }^{*} p<0.05,{ }^{* *} p<0.01,{ }^{* * * *} p<0.0001$ by two-tailed 1412 Fisher's exact test. The number on the bars indicates the number of animals 1413 scored. Black asterisks indicate comparison between each group with the wild1414 type control. Blue asterisks indicate comparison between two specific groups 1415 (highlighted with brackets).

1416 Scale bar (in B for B-E), $1 \mu \mathrm{m}$. 
A

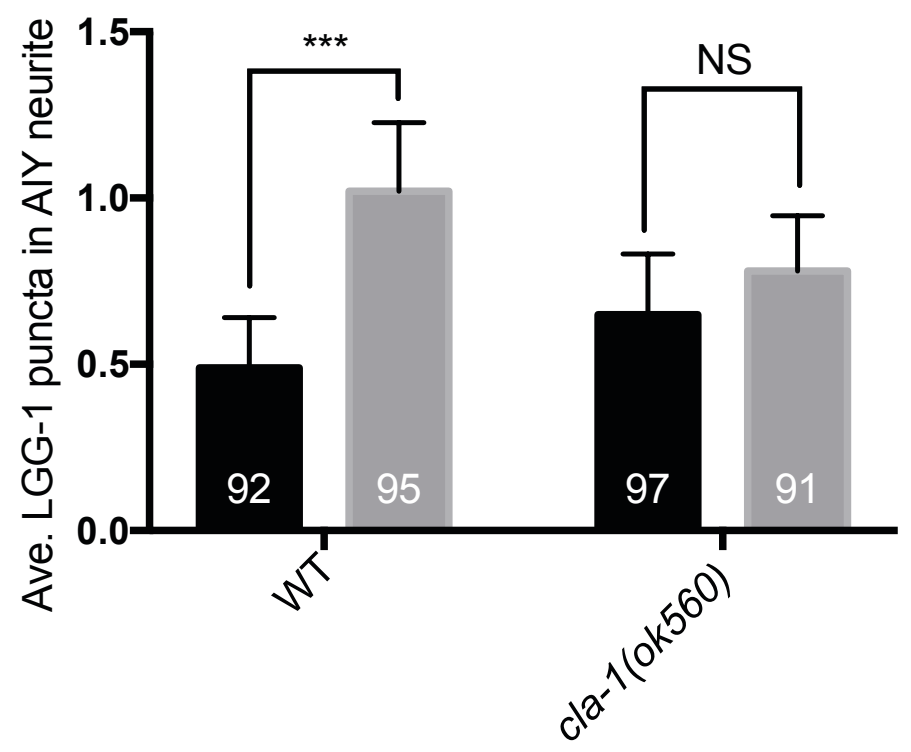

B

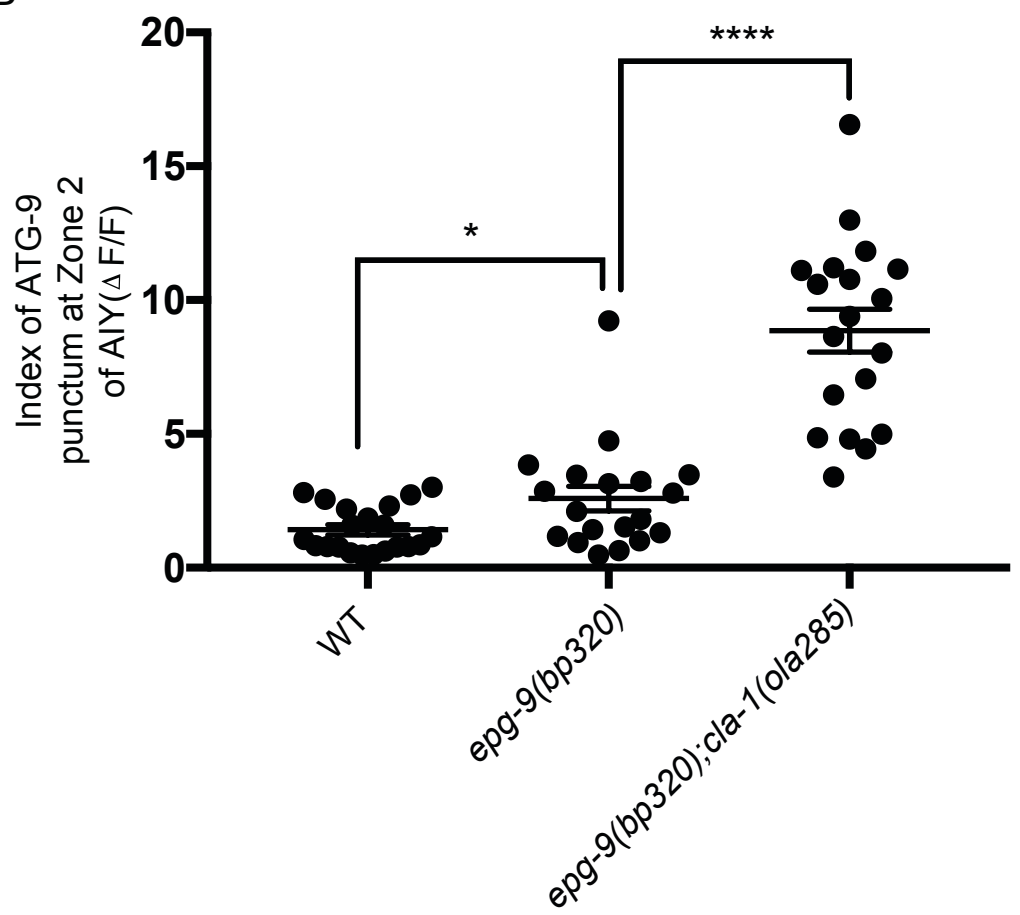

Supplemental figure 5 
1418 Supplemental figure 5. Disrupted ATG-9 trafficking in cla-1(L) mutants is

1419 associated with a deficit in activity-induced autophagosome formation.

1420 (A) Quantification of the average number of LGG-1 puncta in the AIY neurites at $142120^{\circ} \mathrm{C}$ and at $25^{\circ} \mathrm{C}$ for 4 hours in wild type (WT) and cla-1(ok560) mutants. Error

1422 bars represent $95 \%$ confidence interval. "NS" (not significant), ${ }^{* * *}<0.001$ by

1423 Kruskal-Wallis test with Dunn's multiple comparisons test. The number on the bars

1424 indicates the number of animals scored.

1425 (B) Quantification of the index of ATG-9 punctum ( $\Delta F / F)$ at Zone 2 of AIY in the 1426 indicated genotypes. Error bars show standard deviation (SD). ${ }^{*} p<0.05$, $1427^{* * * *} p<0.0001$ by ordinary one-way ANOVA with Tukey's multiple comparison test. 1428 Each dot in the scatter plot represents a single animal. 